\title{
LA MEJORA DEL FACTOR EMPRESARIAL COMO ESTRATEGIA DE COMPETITIVIDAD DE UN MUNICIPIO: EL CASO DE OSUNA
}

\section{THE IMPROVEMENT OF THE BUSINESS FACTOR AS A COMPETITIVENESSSTRATEGYOFAMUNICIPALITY: THE CASE OF OSUNA}

José María Pérez Conde (Escuela Universitaria de Osuna)*

Juan Carlos Morán Álvarez (Universidad de Sevilla) **

Carlos Chavarría Ortiz (Escuela Universitaria de Osuna) * $^{* * *}$

Antonia García Parejo (Escuela Universitaria de Osuna) ${ }^{* * * *}$

\section{RESUMEN}

El objetivo general del estudio es servir de apoyo a las empresas para diseñar un marco analítico e facilite la planificación y ejecución de estrategias empresariales basado en la investigación empírica utilizando el modelo de Rasch. Por otro lado, el propósito específico del análisis es determinar y comparar la posición jerárquica de las empresas de un municipio en relación con la variable latente "fortaleza económica empresarial", así como identificar los factores potenciales más relevantes de la misma, todo ello enfocado a promover el desarrollo económico del municipio sevillano de Osuna.

La medición de la "fortaleza económica empresarial" se apoya en una serie de ítems que la definen, y que el modelo de Rasch clasifica en función de la dificultad o facilidad de logro en la cuantificación realizada. Conocer el posicionamiento de estos ítems en el logro de la variable latente posibilita a las empresas adaptar sus acciones estratégicas, para lograr un mayor impacto. Este análisis puede ser trasladado a otros municipios o bien plantearlo desde una perspectiva multiterritorial y dirigido a actividades productivas similares. Consideramos que este instrumento analítico abre la posibilidad de fortalecer el tejido productivo de un territorio o de una actividad específica, lo que lo convierte en una herramienta muy interesante para mejorar la efectividad y eficiencia en el diseño de estrategias empresariales con el consiguiente efecto en la competitividad y el crecimiento económico del área considerada.

Palabras clave: Modelo de Rasch, estrategia empresarial, municipio, factores de potencialidad, desarrollo territorial.

Códigos JEL: C02, L19, L29, M10, M21.

\section{ABSTRACT}

The general objective of the study is to design a generic analytical framework or framework for the planning and execution of business strategies based on empirical research using the Rasch model. On the other hand, the specific objective of the analysis is to determine and compare the hierarchical position of the companies of a municipality in relation to the latent variable

\footnotetext{
* Autor de correspondencia. José María Pérez Conde, Correo-e: jmperez@euosuna.org

**Correo-e: jcmoran@us.es

***Correo-e: cchavarria@euosuna.org

****Correo-e: directora@euosuna.org

Fecha de envío: 21/01/2021 Fecha de aceptación: 22/04/2021
} 
economic strength, as well as the most relevant potential factors of the same, all focused on fostering and promoting the economic development of the considered municipality.

By obtaining which items are those that most affect the economic strength of the business fabric, these companies can achieve greater progress adapting their strategic actions. Among the options, some strategies will have a higher impact and others lower, all depending on the company in question.

Through this analytical apparatus one could analyze the hierarchy and evolution of the companies of any territory in any selected reference scenario, their possibilities of improvement in relation to those in the first positions, as well as the main measures or facilitating factors of such progress. Therefore, this approach can be a very interesting tool to improve the effectiveness and efficiency in the design of business strategies with the consequent effect on competitiveness and economic development for the area under consideration.

Keywords: Rasch model, business strategy, municipality, potentiality factors, territorial development.

JEL Code: C02, L19, L29, M10, M21.

\section{INTRODUCCIÓN}

En este artículo se van a determinar los factores que más inciden en la fortaleza económica del factor empresarial del municipio de Osuna, situado en la provincia de Sevilla (Andalucía), concretamente en su Comarca de la Sierra Sur y cuenta con aproximadamente 18.000 habitantes. Su ubicación es privilegiada puesto que se encuentra a pie de la autovía A-92 y, por tanto, relativamente muy bien comunicada con algunas de las principales capitales de provincia (Sevilla, Málaga, Granada...). Su factor empresarial está conformado por pymes, fundamentalmente dedicadas a la agricultura y al sector servicios. Para Osuna, como para cualquier localidad, es importante conocer la fortaleza económica de sus empresas para determinar estrategias de mantenimiento y mejora del tejido productivo con futuro, al tiempo que ayuda a identificar ámbitos en los que la falta de competitividad y las dificultades de mejora hacen recomendable dejar de apostar por las empresas que lo conforman. El primer problema que surge es el de la delimitación de la variable latente seleccionada, fortaleza económica empresarial, y el método utilizado para medirla.

Pero más importante que medir el grado de la fortaleza económica del factor empresarial de un municipio, es averiguar cuáles son los factores que más inciden en los resultados y poder determinar en qué línea se deben concretar los progresos y la mejoría en la estrategia empresarial de los municipios para el diseño y planificación de las políticas públicas de dicho territorio.

En este artículo, partiendo de 137 empresas de la ciudad de Osuna y de sus datos extraídos del Sistema de Análisis de Balances Ibéricos, se va a elaborar una clasificación de las mismas según su fortaleza económica empresarial, elegida como variable latente a medir; así como estudiar cuáles son los ítems que más inciden tanto en la medida individual como en el resultado global. Se trata de determinar cómo los factores seleccionados inciden en los niveles de fortaleza económica empresarial, de modo que permitan desvelar las claves de por qué una empresa ocupa esa posición y como la podría mejorar. En definitiva, se pretende marcar las directrices que deben seguir las diferentes empresas para mejorar su posición y fortaleza económica, erigiéndose dentro de los recursos endógenos del territorio considerado como un factor de competitividad del mismo. Dicho estudio, tal y como ya se ha citado en las líneas anteriores, puede contribuir en el diseño y planificación de las políticas públicas de dicho territorio, las cuales podrían servir de coadyuvante para conseguir el objetivo anteriormente planteado: convertir el factor empresarial en un elemento fundamental de competitividad del 
territorio. Así, los objetivos fundamentales a conseguir a partir de la información desprendida de esta medición serían dos:

1. Determinar la posición jerárquica de las empresas según su fortaleza económica, estudiándose los puntos débiles y, sobre todo, los puntos fuertes de aquellas situadas en las primeras posiciones en comparación con las ubicadas en los últimos puestos. Esto permitirá a cada empresa poder modificar o reforzar sus propias estrategias empresariales de cara al futuro.

2. Ordenar, según la importancia de su incidencia diferencial en el constructo o variable latente, los factores de éxito que condicionan la posición, según su fortaleza económica, de las empresas del municipio de Osuna. Esto permitirá a las autoridades tomar decisiones de apoyo al tejido productivo existente.

Para alcanzar los objetivos planteados en el estudio se ha utilizado a nivel metodológico el modelo desarrollado por el matemático danés George Rasch que le da nombre como la herramienta de medida de variables latentes que, además, posibilita valorar el ajuste de las respuestas a los ítems y el que se produce entre la respuesta de un sujeto y los patrones de respuesta de la muestra a la que éste pertenece (Febles, 2008, p.17). Se aplicó por primera vez para la medición de la inteligencia de los soldados daneses y ha sido ampliamente empleado en Psicometría, extendiéndose posteriormente su uso también a otros ámbitos, como el educativo y el económico (Badenes, 2009). Perline et al. (1979) han probado que los modelos que están incluidos en la familia identificada por Rasch son los únicos compatibles con los principios de la teoría de la medida conjunta en el caso probabilístico. Este hecho otorga un papel crucial a los modelos de medida de Rasch en la metodología aplicada en las ciencias sociales (Cliff, 1992; Michell, 1999), siendo empleados en psicología, educación, economía aplicada, salud pública, etc. Un ejemplo reciente de su empleo en el ámbito del análisis de empresas es el artículo de Sánchez-Torné et al. (2020) en el que se cuantifica la Responsabilidad Social Corporativa para empresas en base a un conjunto de ítems. Otro ejemplo claro sería Pérez-Conde et al. (2020) en el cual se analizan los puntos fuertes y los puntos débiles de una muestra de municipios, jerarquizando la posición de los mismos en un continuo lineal y poniendo de manifiesto las posibilidades de mejora de los mismos.

Son muchos los fenómenos económicos y, en general, de las Ciencias Sociales, de difícil medición directa mediante una escala estandarizada y objetiva y para lo que la aplicación de Rasch se ofrece como un instrumento útil, pues a partir de datos categóricos consigue dar respuesta a este problema, delimitando unidades de intervalo que confirman los requisitos de la medición

Por último, reseñar que el presente trabajo consta de la siguiente estructura: revisión de la literatura; metodología; resultados empíricos; conclusiones y referencias bibliográficas.

\section{JUSTIFICACIÓN: REVISIÓN DE LA LITERATURA}

En el presente apartado se pretende poner de manifiesto a través de la literatura existente aquellas cuestiones que constituyen la semilla de este artículo.

Stimson (2016) considera que los científicos regionalistas se han enfrentado a numerosos desafíos en el desarrollo del ámbito interdisciplinario en el que analizan el contexto espacial de los fenómenos sociales, económicos y medioambientales, y asimismo tratar con amplias bases de datos plantea desafíos metodológicos considerables. Dicho artículo estudia la evolución de la Ciencia Regional, las críticas que ha recibido y los retos con los que se ha enfrentado. Analiza 
específicamente, entre otros, algunos retos contemporáneos relacionados con problemas metodológicos como de qué forma medir y los logros del modelo de crecimiento endógeno regional; la necesidad de realizar un mayor uso de microdatos o datos por unidad y de integrarlos en estructuras espaciales más generales... En el presente trabajo también se ha presentado dicha problemática, la cual se ha superado con la aplicación de la metodología de Rasch.

Sili (2018) establece que la acción territorial constituye el insumo básico en la construcción de territorios y del desarrollo territorial. Este concepto permite sustentar el análisis de las formas como la acción pública, la acción colectiva y la acción privada construyen los territorios, muchas veces en forma contradictoria y conflictiva. De esta manera, el concepto de acción territorial constituye un desafío intelectual, pues permite analizar las realidades territoriales replanteando el paradigma de la modernidad que propone que el destino ineludible de los territorios es el desarrollo (modernización y crecimiento), y abre las puertas a pensar en múltiples y diversos caminos para el futuro de los territorios. Este artículo brinda, finalmente, como producto, ideas y propuestas metodológicas para el análisis de la acción territorial.

Nunes y Karnopp (2015) analizan las potencialidades de desarrollo endógeno de Julio de Castilhos, localizado en la Región Central del Estado de Río Grande del Sur en Brasil, con menos de 50 mil habitantes y con base económica en el sector primario. El problema que se investiga es delimitar cuáles son las potencialidades endógenas de desarrollo de Julio de Castilhos - RS, utilizándose metodológicamente un enfoque deductivo, defendiéndose un posicionamiento estratégico para aprovechar las oportunidades locales y regionales.

Torrejón et al. (1996) analizan los factores de competitividad de una población de pequeñas y medianas empresas con una fuerte vinculación sectorial y territorial. El caso de estudio está basado en el principal territorio de especialización textil de la comunidad valenciana, a partir de la información obtenida de una muestra de empresas textiles. Los resultados obtenidos muestran que la adaptación a los requerimientos del cliente es la principal ventaja competitiva en los mercados donde compiten las empresas estudiadas. También se destaca la diversidad de trayectorias empresariales existentes, basadas en el desarrollo de diferentes competencias tecnoproductivas, gerenciales y comerciales.

Subiabre y Chacur (2006) establecen como requisito esencial para que los países, regiones y empresas se inserten con éxito en los flujos de comercio internacional es que sean capaces de acrecentar su competitividad, de una manera duradera y sostenible. Sobre esta premisa, el artículo aborda la forma en que las empresas pueden aportar a la capacidad competitiva territorial, a través de su propio conjunto de estrategias. Para ello, se estudia el caso del sector forestal maderero de la Región del Bío Bío en Chile, por estar señalado por su competitividad e inserción internacional, en conjunto con múltiples factores de entorno que han propiciado y le dan soporte competitivo. En esta base, el artículo se plantea la forma en que las empresas pueden aportar a la gestación de mayores grados de competitividad territorial, haciendo referencia al efecto de las estrategias empresariales sobre el entorno. Se trata la competitividad del sector, analizando los incentivos competitivos que han determinado su inserción en los mercados externos. Se evalúa el grado en que el entorno ha propiciado sus resultados competitivos y se profundiza en las estrategias que las empresas han seguido para incrementar su competitividad, a partir de su interrelación con el entorno. Sobre esto se identifican eslabones ausentes en la estrategia empresarial y se diseñan lineamientos estratégicos propositivos que, con pertinencia al territorio, permitirían a las empresas incrementar su potencial de desarrollo sobre la base del fomento de la competitividad territorial.

Melgarejo et al. (2013) tienen como objetivo determinar si existe alguna relación entre la mipyme y el nivel de competitividad en una región. Para esto se desarrolla el tema 
de la competitividad empresarial con énfasis en el enfoque sistémico de la competitividad. Posteriormente, se realiza una recolección de datos de los indicadores del año 2010, tanto de la competitividad de la mipyme como de la competitividad regional. Por último, se realiza un análisis descriptivo y un análisis correlacional con lo que fue posible obtener los hallazgos y las conclusiones de esta investigación. El principal hallazgo de esta investigación consiste en que existe una relación importante entre el número de mipymes colombianas y la infraestructura educacional y el Producto Interno Bruto, lo cual estaría indicando la importancia de la permanencia de la mipyme para el desarrollo de una región.

Escalona et al. (2011) se centran en dos áreas rurales aragonesas afectadas por procesos de reestructuración en sectores clave de sus economías: la Denominación de Origen vitivinícola de Calatayud y el valle del río Aranda, especializado en la industria del calzado. Una vez presentada la problemática de ambas áreas, se analizan y comparan las diferentes estrategias empresariales. La teoría de las convenciones y el nuevo paradigma de desarrollo rural constituyen el marco teórico de dicho análisis y aportan los elementos para una encuesta sobre la trayectoria empresarial reciente y sus implicaciones para el desarrollo de ambos territorios. Los resultados muestran, con matices, la decidida apuesta de las empresas por la innovación, la calidad y la internacionalización, así como las demandas de las empresas para la mejora de su situación en el territorio. El artículo concluye con diversas propuestas para diseñar políticas adecuadas a los actuales retos de las áreas rurales.

Vázquez Barquero (2012), a diferencia de las interpretaciones macroeconómicas de la crisis de la economía española, fija su atención en el papel que juegan las empresas y territorios. Argumenta que los factores estratégicos para la recuperación económica residen en la capacidad emprendedora existente en los espacios innovadores, en la combinación de las medidas macroeconómicas con las políticas para la innovación y las estrategias empresariales, y en su instrumentación en función del potencial de desarrollo de cada territorio, todo ello contribuiría a la recuperación de la economía y al bienestar social.

Galindo Pérez de Azpillaga (2008) analiza como el aprovechamiento de los posibles sistemas territoriales en los espacios desfavorecidos tiene que estar apoyado en su articulación productiva y que ésta, a su vez esté caracterizada por las iniciativas locales (micro-economía) y el papel que juegan las empresas de cualquier tipo (privadas, públicas, mixtas, cooperativas, etc.) y los empresarios en cada una de ellas. Estos recursos económicos y productivos, unidades encargadas de organizar los factores productivos, se convierten en piezas fundamentales para lograr el crecimiento económico y la generación de empleo. El conocimiento detallado del tejido local de empresas, esto es, el conjunto de empresas y sus relaciones o los eslabonamientos productivos, resultan fundamentales para diseñar una estrategia de fomento productivo local. La falta de información sobre estos aspectos decisivos constituye una dificultad importante para el despegue de los procesos de desarrollo territorial. Aquí se ejemplariza en el caso de la Vega Media de Sevilla, resaltándose la posibilidad de establecer estrategias de sostenibilidad territorial, en una zona de contacto urbano-rural como es ésta, en función al aprovechamiento de los sistemas socio-productivos locales.

Silva (2005) argumenta que en un mundo cada vez más globalizado los gobiernos locales y regionales de América Latina deben asumir nuevos desafíos, entre ellos los de crear o mejorar capacidades competitivas y transformar los sistemas productivos locales. Estos dos aspectos deben vincularse a las políticas territoriales y, más precisamente, al desarrollo de una cultura territorial que integre a ambos. Si bien es cierto que son las empresas las que compiten, su capacidad de competir se puede ver reforzada si el entorno territorial facilita esta dinámica y si, por su parte, ellas también sienten la importancia de ser empresas "del territorio" más que 
empresas "en el territorio". Esta aspiración, sin embargo, choca con la existencia de territorios desigualmente preparados para enfrentar estos desafíos, lo que aconseja distintos tipos de intervención en términos de políticas públicas locales y regionales tendientes a mejorar sus capacidades competitivas.

Argoti (2009) presenta las principales características de las pymes del subsector agroindustrial y su aporte al desarrollo del Departamento de Nariño (Colombia) como factores de generación de crecimiento económico y mejoramiento del bienestar de la población. Se hace referencia a que la competitividad regional crea las condiciones del territorio como un entorno favorable para que las empresas crezcan. Para las pymes agroindustriales de Nariño se establece que la competitividad regional debe constituir una red de actores, factores y recursos que garanticen una adecuada territorialidad para apostar por el crecimiento económico y el bienestar social. El estudio, asumió el criterio cuantitativo para aproximarse de forma más adecuada al análisis de la capacidad competitiva de las pymes del subsector agroindustrial como generadoras del desarrollo del Departamento de Nariño, así mismo la investigación fue de carácter empírica y aplicada, en razón a que persiguió fines de tipo práctico para el Departamento. Considerando el nivel de conocimiento alcanzado, la investigación fue descriptiva y explicativa. De esta manera y a través de encuestas aplicadas a empresarios y expertos, se concluye que las empresas pymes del subsector agroindustrial carecen de la capacidad competitiva para dinamizar el desarrollo del Departamento de Nariño.

Plaza y Blanco (2015) plantean como objetivo establecer estrategias para lograr insertar a las pymes agrícolas en el desarrollo económico local que les permita como organizaciones crecer y mantenerse en un mercado altamente competitivo, la metodología aplicada con el propósito de impulsar el desarrollo de las 317 pymes agrícolas de la provincia de Los Ríos -Ecuador, se basó en talleres, mesas de dialogo, lluvias de ideas en la que participaron los actores involucrados el Gobierno Autónomo descentralizado, la Universidad, los empresarios de las Pymes agrícolas, ciudadanos y juntas parroquiales. Se concluye que las Pymes agrícolas necesitan asesoramiento en el manejo de la gestión administrativa, en la comercialización y mercadeo de los productos, se debe impulsar y fomentar sistemas de financiamiento, programas pilotos de emprendimientos, implementación de asociatividad e implementación de agroindustrias limpias que elaboren productos con valor agregado en el área agrícola.

Sobrino (2005) señala que el estudio de la competitividad territorial requiere metodologías distintas respecto al análisis de la competitividad empresarial, no sólo por los distintos propósitos e instrumentos de la estrategia competitiva, sino también por el posible impacto del desempeño económico de un territorio en el bienestar de su población y por las funciones que asumen los gobiernos central y locales en el fomento y la promoción económica. En este documento se describen y discuten las metodologías de análisis y la medición de la competitividad para distintas escalas territoriales: ciudad, región y país.

Tras la exposición de esta línea de artículos, de este mapa de ideas que ha inspirado gran parte del presente trabajo, cabe resaltar que el denominador común de todos ellos es afrontar con una metodología adecuada (en nuestro caso el modelo de Rasch, el cual se analizará en el siguiente apartado) la importancia de la vinculación entre territorio y factor empresarial, cada uno de ellos evidentemente con sus condicionantes y características particulares. Se trata de considerar el factor empresarial como un recurso endógeno fundamental y por ello es importante diseñar mecanismos como el que se articula en el presente trabajo para tratar de potenciarlo con el consiguiente efecto sobre la competitividad del territorio, así como sobre el nivel de bienestar de su población en términos de crecimiento, empleo y riqueza. Este punto de vista adquiere aún más relevancia en un entorno de Unión Económica y Monetaria donde las políticas macroeconómicas están muy limitadas y solo quedan las políticas microeconómicas. 


\section{METODOLOGÍA}

Este artículo pretende obtener una cuantificación a través de la técnica de Rasch (Rasch, 1980) del constructo o variable latente "Fortaleza económica del factor empresarial del municipio", que vamos a definir a través de diversos ítems, que son considerados como definidores de la fortaleza económica empresarial según criterios financieros, dado que recogen elementos no correlacionados que otorgan un elemento esencial para la definición financiera de la fortaleza de una empresa: rentabilidad económica y la financiera, liquidez general, endeudamiento, cifra de negocio y fondo de maniobra. La bondad de estos factores para definir la fortaleza o debilidad económica empresarial radica en que así lo entiende el registro mercantil en sus informes sobre la situación de cada empresa registrada; además, estos son los principales elementos en los que se basan los informes de fiscalización empresarial que se dan en el mercado y porque el sistema financiero los marca como elementos esenciales para la determinación de la situación de cada empresa. Los definimos:

- Ítem $n^{o}$ 1: Rentabilidad económica. Se puede definir como la relación entre el beneficio antes de intereses e impuestos (beneficio bruto o de explotación) y el activo total. $\mathrm{Su}$ relevancia radica en que representa una medida de eficiencia que se obtiene mediante el desarrollo de la actividad de la empresa a partir de la inversión realizada. Cuanto más elevada sea esta ratio, mejor, porque indicará que se obtiene más productividad del activo.

- Ítem $n^{\circ}$ 2: Rentabilidad financiera. Se puede definir como la relación entre el beneficio neto y los capitales propios (neto patrimonial). Su relevancia en la medición de la fortaleza económica de la empresa radica en que representa el rendimiento obtenido por los capitales propios en un periodo de tiempo, generalmente con independencia de la distribución del resultado. Si la rentabilidad financiera es insuficiente supone una limitación a nuevos fondos propios tanto porque es indicativo de los fondos generados internamente por la empresa como porque restringe la financiación externa. La rentabilidad financiera es, para las empresas con ánimo de lucro, la ratio más importante, ya que mide el beneficio neto generado en relación a la inversión de los propietarios de la empresa. A medida que el valor de la ratio de rentabilidad financiera sea mayor, mejor será esta.

- Ítem $n^{o}$ 3: Liquidez general. La relevancia de la ratio en la variable latente radica en que mide su solvencia, la capacidad que tiene una empresa de poder hacer frente a los pagos futuros a corto plazo. Se calcula tomando el activo corriente (activo circulante), dividiéndolo por el pasivo corriente (exigible a corto plazo). Si el resultado es superior a 100, esto indica que los fondos permanentes están financiando el activo ordinario de la empresa, pero también una elevada ratio de liquidez puede ser indicativa de activos ociosos lo que conlleva una pérdida de rentabilidad. Por el contrario, cabe pensar que si dicha ratio es inferior a 100 la empresa puede tener problemas de liquidez, aunque hay que matizar que esto depende del sector en el que opere la compañía y de las particularidades de éste último.

- Ítem $n^{o}$ 4: Endeudamiento. Esta ratio mide el apalancamiento financiero; es decir, la proporción de deuda que soporta una empresa frente a sus recursos propios. Su relevancia se encuentra en que se calcula a partir de todas las deudas que ha contraído la sociedad tanto a corto como a largo plazo (pasivo), dividiendo dicho valor por el pasivo total más patrimonio neto. Si la ratio de endeudamiento arroja un resultado mayor al 60 por cien significa que la empresa está soportando un excesivo volumen de deuda y la situación puede conllevar la descapitalización de la sociedad y, por tanto, una pérdida de autonomía frente a terceros. 
- Ítem $n^{o}$ 5: Importe neto de cifra de ventas. Su relevancia deriva de que refleja los importes de la venta de los productos y de la prestación de servicios correspondientes a las actividades ordinarias de la sociedad deducidas las bonificaciones y demás reducciones sobre las ventas, así como el Impuesto sobre el Valor Añadido y otros impuestos directamente relacionados con la mencionada cifra de negocios.

- Ítem $n^{o}$ 6: Fondo de maniobra. La relevancia está representada por su papel como definidor de la solvencia de una empresa en el corto plazo. Se calcula sumando el patrimonio neto y el pasivo no corriente y restándole el activo no corriente. Si se presenta un fondo de maniobra positivo, significa que hay recursos financieros a largo plazo suficientes para financiera la inversión a largo plazo.

Para lograr los objetivos que nos hemos marcado, se ha utilizado el programa informático Winsteps versión 3.81.0. La base de datos para el estudio se ha extraído del Sistema de Análisis de Balances Ibéricos (SABI), recopilándose la información de las últimas cuentas presentadas por cada entidad empresarial, es decir, los datos disponibles de las cuentas presentadas en el Registro Mercantil de forma más reciente por parte de cada empresa del municipio de Osuna. De este modo, se obtienen inicialmente 270 empresas. Empero, tras eliminar las empresas extinguidas o en proceso de liquidación ( 29 empresas) así como aquellas para las que no se disponen de datos en más de un ítem o presentan valores excesivamente anómalos situados en los extremos para alguno o algunos de los ítems y que como consecuencia introducirían desajustes que generarían distorsiones importantes en el conjunto del sistema de medición (104 empresas), quedan para el estudio un total de 137 empresas, cuyos nombres y orden de entrada aparecen en la tabla A1 del anexo.

De estas 137 empresas de la tabla citada, 15 presentaron sus cuentas por última vez en el año 2011, 11 empresas lo hicieron en el ejercicio 2012, 110 en 2013 y una en el año 2014. Se considera que la realidad económica empresarial de crisis de estos 4 años es la misma y, por tanto, que los datos utilizados son de utilidad para conseguir los objetivos anteriormente citados.

Una vez obtenidos los datos, el paso siguiente fue hallar el máximo y el mínimo de los valores de cada ítem y establecer un criterio de valoración que convirtiera los datos extraídos en valores recogidos en un intervalo de 1 a 10 con el objetivo de estandarizarlos. Así, para llevar a cabo la categorización de los ítems se calculan 10 tramos equidistantes para cada ítem, asignándose la categoría 1 al intervalo o tramo que contiene los valores más bajos del ítem, y la 10 al intervalo o tramo que contiene los valores más elevados del ítem. En este sentido, se ha de señalar que uno de los ítems elegidos, Endeudamiento, presenta una polaridad invertida; es decir, que un mayor valor del mismo, indica un peor nivel de fortaleza económica del factor empresarial del municipio. Evidentemente esto hace necesario invertir la categorización de dicho ítem, concediéndose la categoría 1 al intervalo o tramo que contiene los valores más altos del ítem, y la 10 al intervalo o tramo que contiene los valores más bajos del ítem.

De este modo, el modelo permite ubicar a las empresas y factores de fortaleza económica en una escala lineal unidimensional representativa del constructo teórico que posibilite contrastar resultados entre las empresas analizadas y la relevancia de los ítems. Por tanto, el modelo propicia el poder llevar a cabo un estudio detallado del factor empresarial del municipio y de los atributos elegidos ya que estos se ubican en un mismo continuo lineal de forma simultánea, apareciendo, por tanto, en dicha línea de manera conjunta las empresas seleccionadas y los factores de fortaleza económica de las mismas, determinándose una sola dirección en la que se considera que el extremo superior e inferior de la línea representan "alto impacto" y "bajo", respectivamente, de la variable en cuestión, consiguiéndose así una jerarquía que llevará a la 
relación que se deriva de las empresas y su nivel de fortaleza económica, así como a la de la relevancia de los factores determinantes del mismo.

El atributo estadístico que diferencia al modelo de Rasch es que los parámetros empresas e ítems están algebraicamente separados y dan lugar a estadísticos suficientes (Andersen, 1973; Masters y Wright, 1984). Rasch considera que, si los ítems elegidos son los correctos, surgirán pocos errores, por lo que es razonable representar la distribución de los errores de lectura mediante una función de probabilidad de Poisson (multiplicativa). La elección de este modelo de Poisson multiplicativo se debía a las propiedades que le cualifican como un modelo de medida (Lord, 1953).

El estudio de los atributos que debían verificar las medidas, llevan a Rasch a la utilización de modelos aditivos exponenciales (modelos de medida), pasando del modelo de Poisson multiplicativo al modelo logístico. Rasch desarrolló su modelo dentro de la teoría de la medición conjunta, que parte de la consideración de que las medidas (observables) fundamentales tienen estructura aditiva.

Siguiendo a Alvarado y Santisteban (2006, p. 10), el instrumento de medida propuesto por Rasch supone tanto la posibilidad de obtener estimadores suficientes de los parámetros, con medidas directas, y haciendo uso de las propiedades de las distribuciones y de los procesos de Poisson compuestos, como la de verificar los requisitos teóricamente exigibles: la estabilidad, la separabilidad, la independencia muestral, así como el requisito imprescindible de aditividad conjunta. Se convierte así en una herramienta de medida de variables latentes que, además, posibilita valorar el ajuste de la categoría alcanzada por cada ítem al conjunto de los mismos, así como el que se produce entre el valor que presenta cada empresa y los patrones de valor de la muestra a la que éste pertenece (Febles, 2008, p. 17).

Esta técnica presenta planteamientos sencillos, pues el valor logrado en un ítem depende tanto del comportamiento, preparación y competencia de la empresa como de la dificultad de conseguir un valor adecuado en el ítem (Febles, 2008. P. 17). Así, según Sánchez y Blanco (2012. p. 37-38), ofrece las siguientes ventajas: es capaz de extraer conclusiones fiables a partir de datos particulares o muestras pequeñas (suficiencia estadística); identifica casos que presentan desajustes respecto al conjunto de los datos, lo que lo diferencia de otras técnicas donde los posibles desajustes son eliminados del análisis por considerar que desvirtúan la muestra (Rasch los identifica para tratar de encontrar una justificación a dicha anomalía, pues suele ser muy clarificador en el caso de hechos o fenómenos económicos); estudia la fiabilidad de los sujetos y de los ítems de forma individual; calcula la fiabilidad y la validez global de las medidas; comprueba la hipótesis de unidimensionalidad; analiza las categorías de cada empresa en cada ítem, jerarquizando estos en función de su dificultad y a las empresas en función de su habilidad; identifica distintos grupos de empresas enfrentando las mediciones con otras variables diferenciales como el tamaño, la actividad... e identifica los puntos fuertes y débiles de cada sujeto de forma individual.

En definitiva, la Quantum Measurement Technique, basada en la Probabilidad de Rasch, es pues, un instrumento de trabajo que permite obtener la medida de una variable latente, siendo una medida más adecuada que otras pues reduce matrices complejas de datos a una variable unidimensional (Álvarez y Martínez, 2001, p. 196).

El resultado final es una medida de la fortaleza económica de cada empresa y de la importancia de cada ítem en ello, así como los desajustes tanto para unas como para otros.

Como cualquier otra variable latente, la fortaleza económica empresarial puede visualizarse como una línea con una dirección a lo largo de la cual se sitúan los ítems y las empresas. La figura 1 muestra que la empresa $\beta_{0}$ no sobrepasa ningún ítem por lo que estará entre las empresas con mayores debilidades. La empresa $\beta_{1}$ sobrepasa sólo al ítem $\delta_{1}$, la empresa $\beta_{2}$ sobrepasa los 


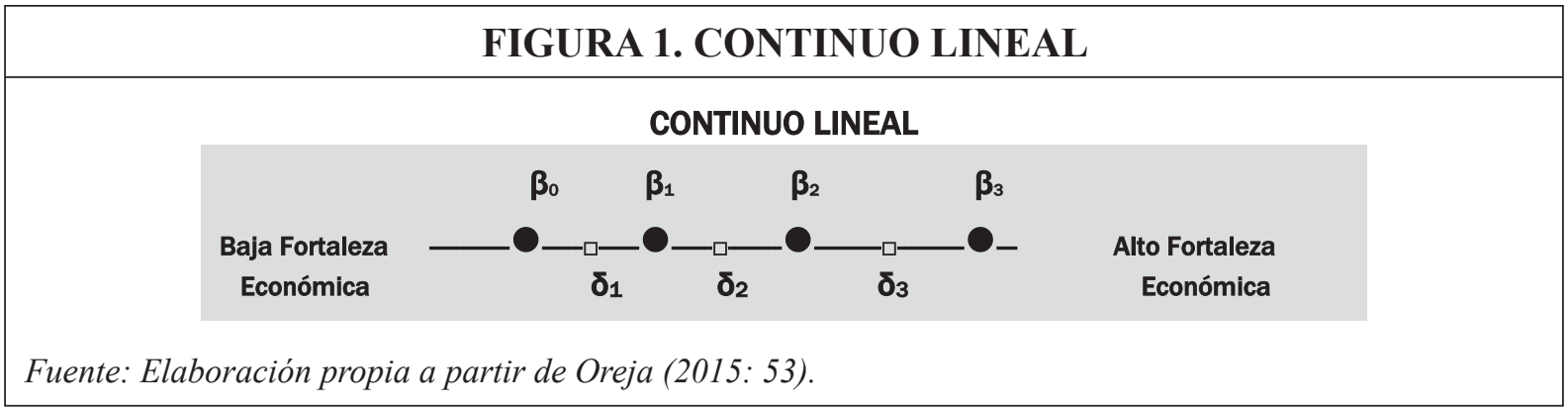

ítems $\delta_{1}$ y $\delta_{2}$, mientras que la empresa $\beta_{3}$ sobrepasa los tres ítems y será la de mayor fortaleza económica. El ítem $\delta_{1}$ es el de menor medida, lo cual representa una fortaleza más fácil de alcanzar y, como consecuencia, un nivel de fortaleza económica más habitual de conseguir, logrado por las empresas $\beta_{1}, \beta_{2}$ y $\beta_{3}$; mientras que el ítem $\delta_{3}$ es el de mayor medida (el que está más a la derecha) y representa un nivel de fortaleza económica más raro, siendo sólo alcanzado por la empresa $\beta_{3}$.

Si se considera $X_{\mathrm{ni}}$ como la variable dicotómica fortaleza económica empresarial municipal que describe el hecho de que una empresa " $n$ " endosa el ítem "i". Si $X_{n i}=1$, entonces la empresa " $n$ " se dice que es potente; por el contrario, si $X_{\text {ni }}=0$ se dice que la empresa " $n$ " no es potente.

Con los cálculos apropiados se obtiene de la fórmula que se presenta, mostrando en el presente caso la probabilidad de que la empresa " $n$ " referida al ítem " $i$ " sea potente, dados los parámetros $\beta_{\mathrm{n}}$ y $\delta_{\mathrm{i}}$. Esta es la fórmula que George Rasch obtuvo en su tratado acerca de las variables latentes (Álvarez y Martínez, 2001, p. 198).

$$
\mathrm{P}\left[\mathrm{X}_{\mathrm{ni}}=1\right] \beta_{\mathrm{n}}, \delta_{\mathrm{i}}=\frac{\mathrm{e}^{(\beta \mathrm{n}-\delta \mathrm{i})}}{1+\mathrm{e}^{(\beta \mathrm{n}-\delta \mathrm{i})}}
$$

Una herramienta de medida configurada con estos ítems, posibilita el poder medir la fortaleza económica de una empresa, $y$, además, permite posicionar a cada empresa estudiada dentro del ámbito de estudio elegido.

Se ha de señalar que el modelo de Rasch es politómico (Rasch, 1980; Andrich, 1978, 1988a y 1988b), de tal modo que en el caso que se está considerando la puntuación asignada se representa por la escala de categorías $\{1,2,3,4,5,6,7,8,9,10\}$ en términos del parámetro sujeto (empresas) e ítem (factores de fortaleza económica de una empresa).

Por tanto, debido a que en el presente trabajo los datos originales de las empresas no aparecen categorizados y toman valores de acuerdo a diferentes escalas y porcentajes, es preciso, conforme al modelo de Rasch de categorías ordenadas de Andrich-Rasch (Andrich, 1978), categorizar de forma conjunta a una misma escala todos los ítems del instrumento de medición diseñado.

El objetivo de esta categorización es transformar varias medidas que representan conceptos de diferente naturaleza o índole, en una medida global que simplifica y permite una interpretación conjunta (Álvarez, 2008).

\section{RESULTADOS EMPÍRICOS}

En la tabla 1 (ampliada con la A2 del anexo) se detalla de manera pormenorizada el posicionamiento jerárquico de las empresas de Osuna a través de la categorización realizada para los 6 ítems ya mencionados. Como se puede ver, las dos primeras posiciones la ocupan 
Compañía Oleícola de Refinación y Envasado SA, y Osuna Mission SL, lo cual es lógico puesto que son dos de las de mayor tamaño del municipio y, además, la producción de ambas se dirige al mercado nacional e internacional y no solo al mercado local o comarcal como en resto de casos con los que trabajamos. En la tabla se encuentran ordenadas de manera decreciente, ocupando los últimos lugares aquellas que obtienen una menor medida $\mathrm{y}$, por tanto, presentan una menor fortaleza económica.

\section{TABLA 1. MEDIDA DE FORTALEZA ECONÓMICA DE LAS EMPRESAS DEL MUNICIPIO DE OSUNA}

\begin{tabular}{|c|c|c|c|c|c|c|c|}
\hline \multirow{2}{*}{$\begin{array}{l}\mathrm{N}^{0} \text { de } \\
\text { entrada }\end{array}$} & \multirow[t]{2}{*}{ Medida } & \multirow{2}{*}{$\begin{array}{c}\text { DS: } \\
\text { Desviac. } \\
\text { Estándar }\end{array}$} & \multicolumn{2}{|c|}{ INFIT } & \multicolumn{2}{|c|}{ OUTFIT } & \multirow[t]{2}{*}{ Empresa } \\
\hline & & & MNSQ & ZSTD & MNSQ & ZSTD & \\
\hline 30 & 0,62 & 0,30 & 7,46 & 4,7 & 9,90 & 4,6 & $\begin{array}{l}\text { Compañía Oleícola de } \\
\text { Refinación y Envasado } \\
\text { SA }\end{array}$ \\
\hline 95 & 0,25 & 0,26 & 4,43 & 4,8 & 5,69 & 4,2 & Osuna Mission SL \\
\hline 69 & 0,06 & 0,26 & 3,53 & 3,7 & 2,82 & 2,4 & $\begin{array}{l}\text { Hacienda la Carrascal } \\
\text { SL }\end{array}$ \\
\hline 130 & $-0,01$ & 0,26 & 5,23 & 4,8 & 4,28 & 3,5 & $\begin{array}{l}\text { Urbaneja y Espinosa } \\
\text { SL }\end{array}$ \\
\hline 97 & $-0,08$ & 0,27 & 1,60 & 1,2 & 1,67 & 1,2 & Osunalia SL \\
\hline 11 & $-0,23$ & 0,29 & 1,31 & 0,7 & 1,23 & 0,5 & Alvaro Espuny SL \\
\hline 86 & $-0,23$ & 0,29 & 2,06 & 1,6 & 2,03 & 1,4 & Madre Carmen SL \\
\hline 129 & $-0,32$ & 0,30 & 2,12 & 1,5 & 1,42 & 0,8 & Ufacon SL \\
\hline 55 & $-2,09$ & 0,35 & 4,35 & 3,3 & 2,70 & 1,3 & $\begin{array}{l}\text { Empresarial Morón } \\
\text { Activa SL }\end{array}$ \\
\hline 77 & $-2,09$ & 0,35 & 1,89 & 1,4 & 3,07 & 1,4 & $\begin{array}{l}\text { Inversiones Carmona } \\
\text { Bejarano SL }\end{array}$ \\
\hline 93 & $-2,09$ & 0,35 & 2,81 & 2,2 & 2,81 & 1,3 & $\begin{array}{l}\text { Núñez Taller } \\
\text { Mecánico SL }\end{array}$ \\
\hline 126 & $-2,09$ & 0,35 & 1,13 & 0,4 & 0,46 & 0,0 & Todogangas Urso SL \\
\hline 8 & $-2,22$ & 0,35 & 1,43 & 0,8 & 0,75 & 0,3 & Albarizuela $2007 \mathrm{SL}$ \\
\hline 50 & $-2,22$ & 0,35 & 4,26 & 3,3 & 4,39 & 1,8 & $\begin{array}{l}\text { Delgado Ortega } \\
\text { Francisco Antonio } \\
\text { 002375082Z SLNE }\end{array}$ \\
\hline 82 & $-2,22$ & 0,35 & 3,13 & 2,5 & 2,88 & 1,4 & Largui Inmobiliaria SL \\
\hline 87 & $-2,22$ & 0,35 & 1,75 & 1,2 & 0,70 & 0,2 & Mancarde SL \\
\hline 134 & $-2,34$ & 0,37 & 3,64 & 2,6 & 2,81 & 1,4 & $\begin{array}{l}\text { Urso Sistemas } \\
\text { Informáticos SL }\end{array}$ \\
\hline MEAN & $-1,11$ & 0,40 & 0,84 & $-0,3$ & 0,76 & 0,0 & \\
\hline S.D. & 0,52 & 0,04 & 1,27 & 1,4 & 1,29 & 1,0 & \\
\hline
\end{tabular}

Nota: Los posibles desajustes que puedan surgir en la medición son analizados mediante dos estadísticos (Oreja, 2005, p. 40):1 INFIT es un valor sensible al comportamiento inesperado que afecta a los ítems cuya dificultad está cerca del nivel de habilidad de la empresa, y correspondería a un ajuste interno; OUTFIT es un valor sensible al comportamiento inesperado que afecta a los ítems cuya dificultad está lejos del nivel de habilidad de la empresa, y correspondería a un ajuste externo (Tristán, 2001). Abas pueden presentarse como media cuadrática (MNSQ) o como test de hipótesis (ZSTD).

Fuente: Elaboración propia a partir de Winsteps 3.81.0. 
A continuación, se trata de abordar el segundo de los objetivos primordiales de este estudio: ordenar cuáles son los ítems más y menos importantes a la hora de generar un diferencial en la fortaleza económica del factor empresarial del municipio de Osuna.

En la tabla 2 se muestran las condiciones facilitadoras de la fortaleza económica de las empresas de un municipio elegidas para el estudio, ordenadas de menos a más presencia en el entorno seleccionado de acuerdo con la percepción o puntuación de las empresas. Según los valores de esta tabla, los resultados ponen de relieve que el ítem con una medida más alta, y, por tanto, más difícil de superar o conseguir es el Importe neto de cifra de ventas, mientras que el factor con una medida más baja, y, por tanto, más fácil de superar o conseguir es el Endeudamiento.

Por tanto, entre los seleccionados, el factor que más grado de influencia tiene a la hora de generar un diferencial positivo en clave de fortaleza económica para las empresas que alcancen una categoría mayor es el ítem Importe neto de cifra de ventas, con una medida de 1,54 unidades logit, seguido del ítem Liquidez general, con una medida de 1,45. Del mismo modo, el siguiente ítem en importancia en cuanto a incidencia a la hora de marcar un diferencial en términos de fortaleza económica empresarial sería Fondo de maniobra con una medida de 1,08 unidades logit. Esto significaría que dichos ítems son difíciles de lograr, que existen marcadas diferencias entre las empresas y que son pocas las que consiguen alcanzar un nivel categórico importante en estos y que, por tanto, pueden marcar un diferencial importante en la fortaleza económica de las mismas.

\section{TABLA 2. MEDIDA DE LA IMPORTANCIA DE LOS ÍTEMS EN LA MEDIDA DE LA FORTALEZA ECONÓMICA EMPRESARIAL DE LAS EMPRESAS DEL MUNICIPIO DE OSUNA}

\begin{tabular}{|l|c|c|c|c|c|l|}
\hline \multirow{2}{*}{ Medida } & \multirow{2}{*}{$\begin{array}{c}\text { D.S: } \\
\text { Desv. } \\
\end{array}$} & \multicolumn{2}{|c|}{ INFIT } & \multicolumn{2}{c|}{ OUTFIT } & \multirow{2}{*}{ ÍTEM } \\
\cline { 3 - 6 } & Estándar & MNSQ & ZSTD & MNSQ & ZSTD & \\
\hline 1,54 & 0,14 & 1,75 & 1,8 & 0,48 & $-1,1$ & Importe Neto de Cifra de Ventas \\
\hline 1,45 & 0,13 & 2,60 & 3,4 & 0,47 & $-1,2$ & Liquidez General \\
\hline 1,08 & 0,09 & 1,22 & 1,0 & 0,88 & $-0,2$ & Fondo de Maniobra \\
\hline$-0,77$ & 0,07 & 0,60 & $-2,9$ & 0,49 & $-3,4$ & Rentabilidad Económica \\
\hline$-1,12$ & 0,07 & 1,10 & 0,7 & 0,87 & $-0,7$ & Rentabilidad Financiera \\
\hline$-2,18$ & 0,06 & 0,90 & $-1,0$ & 1,40 & 3,0 & Endeudamiento \\
\hline 0,00 & 0,09 & 1,36 & 0,5 & 0,77 & $-0,6$ & MEAN \\
\hline 1,43 & 0,03 & 0,65 & 2,0 & 0,34 & 1,9 & S.D. \\
\hline
\end{tabular}

Fuente: Elaboración propia a partir de Winsteps 3.81.0.

En relación a los ítems capaces de generar menores diferencias entre empresas porque la mayor parte de las mismas alcanzó una categoría elevada en los mismos, se ha de destacar la última posición de la tabla es ocupada por el ítem Endeudamiento con una medida de la fortaleza económica empresarial de -2,18 unidades logit. También se sitúan por debajo de la media de 0,00 unidades logits, la Rentabilidad financiera y Rentabilidad económica con unas medidas ya negativas de $-1,12$ y $-0,77$ unidades logit, respectivamente, lo cual indicaría que su impacto como factores diferenciales en la fortaleza económica empresarial sería bastante menor que el de los tres primeros ítems. Esto último significaría que la mayor parte del sistema empresarial de Osuna se encuentra bastante saneado puesto que una proporción elevada de sujetos alcanza un valor de categorización elevado, es decir, el tejido empresarial está poco endeudado, lo cual es tremendamente positivo de cara a su fortaleza económica. 
Por último, se pueden analizar los mapas de diagnóstico obtenidos para cada una de las empresas y los cuales ayudan a determinar con mayor facilidad qué ítems son considerados como puntos fuertes o débiles. En la tabla 3, se refleja como las empresas con las dos medidas más elevadas en "fortaleza económica empresarial", presentan como puntos fuertes el I5. Importe neto de cifra de ventas y el I6. Fondo de maniobra, que son los que ocupan la primera y tercera posición en dificultad de logro (tabla 2), por lo que presentan una elevada capacidad para generar un diferencial importante en la fortaleza económica empresarial, lo que tiene como consecuencia que estos dos sujetos ocupen las dos primeras posiciones en la ordenación jerárquica de la tabla 1. En cuanto a puntos débiles, destacan a los otros 4 ítems, aunque en orden diferente, pues el modelo entiende que estas dos empresas deberían tener una mayor categoría dados los altos valores en las otras dos que tienen mayor dificultad de logro que estas. Es cierto que una de ellas es el I3. Liquidez general que es segundo con mayor dificultad de logro, por ello el presentar categoría (1) la sitúa como debilidad pero no entre las más altas. Igualmente, las empresas en tercera y cuarta posición presentan un valor muy elevado en este ítem, I3. Liquidez general, lo cual les permite este posicionamiento en el ranking tan elevado, a pesar de las categorías más normales registradas en los demás ítems.

\begin{tabular}{|c|c|c|}
\hline \multicolumn{3}{|c|}{$\begin{array}{c}\text { TABLA 3. ÍTEMS DESTACADOS COMO PUNTOS FUERTES O DÉBILES EN } \\
\text { LAS } 20 \text { EMPRESAS CON MAYOR FORTALEZA ECONÓMICA }\end{array}$} \\
\hline $\begin{array}{l}\text { EMPRESAS SEGÚN ORDEN } \\
\text { DE FORTALEZA }\end{array}$ & PUNTOS FUERTES & PUNTOS DÉBILES \\
\hline $\begin{array}{l}1^{\circ} . \text { Compañía Oleícola de } \\
\text { Refinación y Envasado SA }\end{array}$ & $1^{\circ} \mathrm{ICN}(10)-3^{\circ} \mathrm{FM}(10)$ & $6^{\circ} \mathrm{E}(7)-4^{\circ} \mathrm{RE}(6)-2^{\circ} \mathrm{LG}(1)-5^{\circ} \mathrm{RF}(7)$ \\
\hline $2^{\circ}$. Osuna Mission SL & $3^{\circ} \mathrm{FM}(10)-1^{\circ} \mathrm{ICN}$ & $4^{\circ} \mathrm{RE}(5)-6^{\circ} \mathrm{E}(7)-5^{\circ} \mathrm{RF}(6)-2^{\circ} \mathrm{LG}(1)$ \\
\hline $3^{\circ}$. Hacienda la Carrascal SL & $2^{\circ} \mathrm{LG}(9)$ & $4^{\circ} \mathrm{RE}(5)-5^{\circ} \mathrm{RF}(6)-3^{\circ} \mathrm{FM}(2)-1^{\circ} \mathrm{ICN}(1)$ \\
\hline $4^{\circ}$. Urbaneja y EspinoSA SL & $2^{\circ} \mathrm{LG}(10)$ & $5^{\circ} \mathrm{RF}(5)-4^{\circ} \mathrm{RE}(5)-3^{\circ} \mathrm{FM}(1)-1^{\circ} \mathrm{ICN}(1)$ \\
\hline $5^{\circ}$. Osunalia SL & $4^{\circ} \mathrm{RE}(10)-5^{\circ} \mathrm{RF}(9)$ & $3^{\circ} \mathrm{FM}(1)-2^{\circ} \mathrm{LG}(1)-1^{\circ} \mathrm{ICN}(1)$ \\
\hline $6^{\circ}$. Alvaro Espuny SL & $2^{\circ} \mathrm{LG}(5)$ & $5^{\circ} \mathrm{RF}(6)-3^{\circ} \mathrm{FM}(1)$ \\
\hline $7^{\circ}$. Madre Carmen SL & $2^{\circ} \mathrm{LG}(6)-6^{\circ} \mathrm{E}(10)$ & $4^{\circ} \mathrm{RE}(5)-5^{\circ} \mathrm{RF}(6)-3^{\circ} \mathrm{FM}(1)$ \\
\hline $8^{\circ}$. Ufacon SL & $3^{\circ} \mathrm{FM} \mathrm{(7)}$ & $4^{\circ} \mathrm{RE}(5)-5^{\circ} \mathrm{RF}(6)-6^{\circ} \mathrm{E}(8)-2^{\circ} \mathrm{LG}(1)-1^{\circ} \mathrm{ICN}(1)$ \\
\hline $9^{\circ}$. Dasy Organización SL & $1^{\circ} \mathrm{ICN}(6)$ & $5^{\circ} \mathrm{RF}(5)-4^{\circ} \mathrm{RE}(5)-3^{\circ} \mathrm{FM}(1)$ \\
\hline $10^{\circ}$. Parafarmacia al Galope SL & $4^{\circ} \mathrm{RE}(7)-6^{\circ} \mathrm{E}(10)$ & $5^{\circ} \mathrm{RF}(6)-3^{\circ} \mathrm{FM}(1)$ \\
\hline $11^{\circ}$. Urso SL & $6^{\circ} \mathrm{E}(10)$ & $5^{\circ} \mathrm{RF}(6)$ \\
\hline $\begin{array}{l}12^{\circ} . \text { Agricultura y Servicios el } \\
\text { Aljibón SL }\end{array}$ & $4^{\circ} \mathrm{RE}(7)$ & $6^{\circ} \mathrm{E}(8)$ \\
\hline $13^{\circ}$. Cortijo Carrión SL & $6^{\circ} \mathrm{E}(10)$ & - \\
\hline $14^{\circ}$. Masur Agricola SL & $1^{\circ} \mathrm{ICN}(3)-3^{\circ} \mathrm{FM}(3)$ & $4^{\circ} \mathrm{RE}(5)-6^{\circ} \mathrm{E}(8)$ \\
\hline $15^{\circ}$. SAT Las Tres Palmeras & $6^{\circ} \mathrm{E}(10)-2^{\circ} \mathrm{LG}(2)$ & - \\
\hline $\begin{array}{l}16^{\circ} \text {. Suministros Eléctricos Osuna } \\
\text { SL }\end{array}$ & $6^{\circ} \mathrm{E}(10)-2^{\circ} \mathrm{LG}(2)$ & - \\
\hline $17^{\circ}$. Cemedi Osuna SRL & $6^{\circ} \mathrm{E}(10)$ & - \\
\hline $18^{\circ}$. Cortijo Algarabejo SL & $6^{\circ} \mathrm{E}(10)$ & $4^{\circ} \mathrm{RE}(5)$ \\
\hline $19^{\circ}$. Creaciones Herymat SL & $6^{\circ} \mathrm{E}(10)-2^{\circ} \mathrm{LG}(2)$ & $4^{\circ} \mathrm{RE}(5)$ \\
\hline $20^{\circ}$. Explotación Oxona SL & $3^{\circ} \mathrm{FM}(5)$ & $5^{\circ} \mathrm{RF}(5)-4^{\circ} \mathrm{RE}(5)$ \\
\hline $\begin{array}{l}\text { Nota aclaratoria: } 1^{\circ} \mathrm{ICN}=\text { Importe } \mathrm{Ne} \\
\text { de Maniobra }-4^{\circ} \mathrm{RE}=\text { Rentabilidad } \mathrm{E} \\
\text { (categoría). } \\
\text { Fuente: Elaboración propia a partir }\end{array}$ & $\begin{array}{l}\text { to de Cifra de Ventas - } 2 \\
\text { conómica }-5^{\circ} \mathrm{RF}=\text { Ren } \\
\text { de Winsteps 3.81.0. }\end{array}$ & $\begin{array}{l}\mathrm{LG}=\text { Liquidez General }-3^{\circ} \mathrm{FM}=\text { Fondo } \\
\text { abilidad Financiera }-6^{\circ} \mathrm{E}=\text { Endeudamiento }\end{array}$ \\
\hline
\end{tabular}


Este análisis permite a una empresa local conocer que valores son considerados por el modelo como no esperados, y por ello los sitúa como fortaleza o debilidad. Por ejemplo, $6^{\circ}$. Alvaro Espuny SL debe aprovechar e impulsar la fortaleza destacada en un ítem tan difícil como la liquidez general, con una categoría (5) que sitúa su activo corriente muy por encima de su pasivo corriente, intentando incidir en el incremento relativo del activo corriente. Al mismo tiempo, ha de corregir las razones que lo llevan a presentar comparativamente categorías (6) en Rentabilidad financiera, el segundo de más fácil logro, y categoría (1) en Fondo de Maniobra, el tercero de mayor dificultad. En cuanto a la primera, es una rentabilidad financiera del 20,36 por cien, que no es un mal dato, si bien dada su liquidez general y comparándola con los valores presentados en la matriz del conjunto de valores [empresas x ítems] el modelo lo destaca como debilidad y considera que podría tener un mayor valor con relativa facilidad. El segundo, su fondo de maniobra es positivo, aunque reducido, por lo que la categoría (1) es considerada como muy baja teniendo en cuenta el valor logrado en el resto de los ítems, especialmente en liquidez general.

Se puede hacer un análisis por empresa, especialmente en lo referente a sus debilidades y fortalezas para minimizar las primeras y potenciar las segundas, enfrentando estos resultados a los datos originales y tratando de dar explicación a la debilidad o fortaleza en cuestión.

Para estas 20 primeras empresas del ranking, se ha de destacar que, en relación con los puntos fuertes, el ítem $1^{\circ}$, el de mayor dificultad de logro, aparece como tal en 4 empresas, el ítem $2^{\circ}$ en 7 empresas, el ítem $3^{\circ}$ surge como tal en 5 ocasiones. En cuanto a los de más fácil logro, el ítem $4^{\circ}$ aparece 3 veces, el ítem $5^{\circ}$ se manifiesta 1 vez, y el ítem $6^{\circ}$ aparece como tal en 9 ocasiones. Por tanto, los ítems Endeudamiento y Liquidez general predominan con un rol de fortaleza en las empresas situadas en las primeras posiciones, destacando, sobre todo, la presencia del ítem 3 que ocupa la segunda posición en la tabla 2.

Por otro lado, y en relación con las debilidades presentadas por dichas empresas, se ha de resaltar que el ítem $1^{\circ}$ aparece como tal en 4 empresas; el $2^{\circ}$ también surge 4 veces; el $3^{\circ}$ se manifiesta como tal en 7 ocasiones; el $4^{\circ}$ aparece 11 veces, 7 de las cuales como primera debilidad; el $5^{\circ}$ esta en 11 ocasiones, de las cuales 6 veces como la principal debilidad; y el $6^{\mathrm{o}}$ se manifiesta como tal en 5 empresas. En definitiva, los ítems rentabilidad económica y rentabilidad financiera son los puntos débiles con mayor predominio entre las 20 empresas consideradas, si bien hay que tener presente que 4 de ellas no muestran ninguna debilidad, según el modelo.

Lo mismo podemos hacer con las empresas que se recogen en la tabla 4, las que han obtenido las 20 peores mediciones en Fortaleza Económica Empresarial en Osuna. Este grupo, se caracteriza en contraposición a las 20 primeras empresas del ranking, por no presentar como punto fuerte ninguno de los factores situados en las dos primeras posiciones de la tabla 2, Importe neto de cifra de ventas y Liquidez general, siendo también menor la presencia del situado en tercero en dificultad de logro, Fondo de maniobra, que solo está presente en dos empresas: Prefabricados y Morteros del Sur SL e Inversiones Carmona Bejarano SL. Lógicamente, al tratarse de las empresas con peor medición, las categorías alcanzadas en estos tres son coherentemente bajos, de ahí que ninguno de los tres surja como debilidad, dado que el modelo ya esperaba valores reducidos y los sitúa en un cuadrante de Nivel Bajo Esperado, el cual engloba aquellos que están por encima del nivel de habilidad de estas empresas y para los cuales el modelo no espera una puntuación elevada, como así ha sido en 20 ocasiones para los dos primeros ítems mencionados y 18 veces para el tercero, y ello condena a estas empresas a ocupar las últimas posiciones.

En cuanto a los otros tres, aquellos que requieren de menor capacidad para su logro, como debilidades son señalados el $6^{\circ}$ Endeudamiento en 10 de las 20 empresas como debilidad más 
importante o única; el $5^{\circ}$ Rentabilidad financiera en 8 empresas como debilidad única y el $4^{\circ}$ Rentabilidad económica en 2 ocasiones como segunda debilidad; además, hay dos empresas que no presentan debilidades. En cuanto a las fortalezas, además de los dos casos citados para el $3^{\circ}$ fondo de maniobra, el $4^{\circ}$ rentabilidad económica es señalado en 10 de las empresas, en 6 de las cuales como segunda debilidad; el $5^{\circ}$ Rentabilidad financiera en 10 empresas y el $6^{\circ}$ Endeudamiento en 4 empresas.

\begin{tabular}{|c|c|c|}
\hline \multicolumn{3}{|c|}{$\begin{array}{l}\text { TABLA 4. ÍTEMS DESTACADOS COMO PUNTOS FUERTES O DÉBILES EN } \\
\text { LAS } 20 \text { EMPRESAS CON MENOR FORTALEZA ECONÓMICA }\end{array}$} \\
\hline EMPRESAS SEGÚN ORDEN DE FORTALEZA & PUNTOS FUERTES & PUNTOS DÉBILES \\
\hline $118^{\circ}$. Constru Ramírez Fuentes SL & $6^{\circ} \mathrm{E}(7)$ & $5^{\circ} \mathrm{RF}(4)$ \\
\hline $119^{\circ}$. Importacion Automoviles Alvaro SL & $5^{\circ} \mathrm{RF}(6)$ & $6^{\circ} \mathrm{E}(5)$ \\
\hline $120^{\circ}$. Muebles Antonio Martín SL & $5^{\circ} \mathrm{RF}(8)$ & $6^{\circ} \mathrm{E}(5)-4^{\circ} \mathrm{RE}(3)$ \\
\hline $121^{\circ}$. Osuna Oliva SL & - & - \\
\hline $122^{\circ}$. Rosan Demoliciones SL & $6^{\circ} \mathrm{E}(7)$ & $5^{\circ} \mathrm{RF}(4)$ \\
\hline $123^{\circ}$. Talleres Blancomotor SL & $5^{\circ} \mathrm{RF}(6)$ & $6^{\circ} \mathrm{E}(5)$ \\
\hline $124^{\circ}$. Carpinteria Fadema SRL & $6^{\circ} \mathrm{E}(7)-4^{\circ} \mathrm{RE}(5)$ & $5^{\circ} \mathrm{RF}(3)$ \\
\hline $125^{\circ}$. Danjo Obras y Proyectos SL & $4^{\circ} \mathrm{RE}(5)$ & - \\
\hline $126^{\circ}$. Granja la Dicha SL & $4^{\circ} \mathrm{RE}(9)$ & $6^{\circ} \mathrm{E}(2)$ \\
\hline $127^{\circ}$. Prefabricados y Morteros del Sur SL & $3^{\circ} \mathrm{FM}(2)-4^{\circ} \mathrm{RE}(5)$ & $5^{\circ} \mathrm{RF}(3)$ \\
\hline $128^{\circ}$. Arregui Electrodomésticos SL & $5^{\circ} \mathrm{RF}(6)$ & $6^{\circ} \mathrm{E}(4)$ \\
\hline $129^{\circ}$. EmpreSArial Morón Activa SL & $5^{\circ} \mathrm{RF}(9)$ & $6^{\circ} \mathrm{E}(3)-4^{\circ} \mathrm{RE}(1)$ \\
\hline $130^{\circ}$. Inversiones Carmona Bejarano SL & $3^{\circ} \mathrm{FM}(2)-4^{\circ} \mathrm{RE}(5)$ & $5^{\circ} \mathrm{RF}(1)$ \\
\hline $131^{\circ}$. Núñez Taller Mecánico SL & $5^{\circ} \mathrm{RF}(6)-4^{\circ} \mathrm{RE}(5)$ & $6^{\circ} \mathrm{E}(2)$ \\
\hline $132^{\circ}$. Todogangas Urso SL & $4^{\circ} \mathrm{RE}(5)$ & $5^{\circ} \mathrm{RF}(2)$ \\
\hline $133^{\circ}$. Albarizuela $2007 \mathrm{SL}$ & $6^{\circ} \mathrm{E}(7)-4^{\circ} \mathrm{RE}(4)$ & $5^{\circ} \mathrm{RF}(1)$ \\
\hline $\begin{array}{l}134^{\circ} \text {. Delgado Ortega Francisco Antonio } \\
002375082 Z \text { SLNE }\end{array}$ & $5^{\circ} \mathrm{RF}(6)-4^{\circ} \mathrm{RE}(5)$ & $6^{\circ} \mathrm{E}(1)$ \\
\hline $135^{\circ}$. Largui Inmobiliaria SL & $5^{\circ} \mathrm{RF}(7)$ & $6^{\circ} \mathrm{E}(2)$ \\
\hline $136^{\circ}$. Mancarde SL & $4^{\circ} \mathrm{RE}(5)$ & $5^{\circ} \mathrm{RF}(1)$ \\
\hline $137^{\circ}$. Urso Sistemas Informáticos SL & $5^{\circ} \mathrm{RF}(7)$ & $6^{\circ} \mathrm{E}(2)$ \\
\hline
\end{tabular}

Nota aclaratoria: $1^{\circ} \mathrm{ICN}=$ Importe Neto de Cifra de Ventas $-2^{\circ} \mathrm{LG}=$ Liquidez General $-3^{\circ} \mathrm{FM}=$ Fondo de Maniobra $-4^{\circ} \mathrm{RE}=$ Rentabilidad Económica $-5^{\circ} \mathrm{RF}=$ Rentabilidad Financiera $-6^{\circ} \mathrm{E}=$ Endeudamiento (categoría).

Fuente: Elaboración propia a partir de Winsteps 3.81.0.

\section{CONCLUSIONES}

La presente investigación, basándose en la medición de la fortaleza económica del factor empresarial de Osuna, obtenida mediante la aplicación del modelo de Rasch, ha logrado jerarquizar la posición de las empresas en función de esta variable latente y poner de manifiesto la relevancia o incidencia diferencial de los factores de potencialidad en dicho proceso y, en definitiva, la existencia de asimetrías entre los sujetos considerados, las cuales explican la ordenación de los mismos en base al constructo elegido.

Ello puede ayudar a cada empresa, sin lugar a dudas, al diseño más eficaz y eficiente de estrategias empresariales derivadas de los resultados que se presentan. Así, vemos como la 
empresa con peor valoración puede apoyarse en su única fortaleza, la rentabilidad financiera para conseguir mejorar su posicionamiento, así como mejorar su ratio de endeudamiento que es su debilidad destacada por el modelo, y eso que este lo señala como el ítem en el que resulta más fácil conseguir un valor alto. Establecer mecanismos que reduzcan el peso del endeudamiento y/o aumenten el de recursos propios permitirá a la empresa mejorar su situación de forma importante, en particular la primera opción, porque la segunda presionaría a la baja a la rentabilidad financiera.

Igualmente, la acción política local en Osuna puede trabajar para mejorar las opciones empresariales para incrementar su Importe neto de su cifra de ventas y el activo corriente de las empresas que conforman su tejido empresarial. Es más, la primera contribuiría a mejorar de forma importante la segunda, y con ello el tercer factor en importancia, el fondo de maniobra, así como impactar en los otros tres de forma positiva.

Por tanto, y según todo lo comentado, se debe orientar el diseño tanto de las estrategias empresariales como de las políticas regionales y locales hacia la potenciación de los factores de fortaleza económica empresarial que ocupan las primeras posiciones en la tabla 2, así como evitar valores negativos en ítems de fácil logro como las tres últimas de dicha tabla.

El análisis se desarrolla en un escenario constituido por unos sujetos que presentan datos muy heterogéneos, resultado de la divergencia tanto en las características propias de cada territorio (económicas, sociales, culturales, institucionales...), así como del nivel de competitividad de cada empresa. En este contexto, el presente trabajo de investigación se presenta como un instrumento de análisis funcional, operativo, práctico, flexible, apropiado y eficiente para el estudio económico de las empresas a nivel municipal, pero también podría ser interesante hacerlo a nivel sectorial en un grupo de empresas pertenecientes a una misma actividad productiva, en un ámbito territorial más amplio, lo que dejamos para artículos venideros. Lo cierto es que este tipo de análisis permite jerarquizar a las empresas seleccionadas en función de su fortaleza económica empresarial, posibilitando incluso la ejecución de comparaciones intertemporales, así como ordenar los factores de potencialidad elegidos según su capacidad o relevancia para generar una incidencia diferencial en el citado proceso, siendo este último aspecto muy útil y valioso para la recomendación de estrategias empresariales.

Este trabajo permite identificar los ítems fuertes y débiles en los que destaca cada empresa, lo que enfrentado a la ponderación que experimenta cada uno de estos en la medición global nos facilita identificar cuál puede ser la estrategia específica de cada una de ellas si desea mejorar su fortaleza económica y competitividad, tal y como hemos visto en ejemplos concretos que hemos enunciado.

\section{BIBLIOGRAFÍA}

Alvarado, J. M. y Santisteban, C. (2006). “La validez en la medición psicológica”. Madrid: UNED.

Álvarez, J. C. M. y Martínez, P. Á. (2001). "Medida del desarrollo humano para los países de la América Latina". El Trimestre Económico, 195-208.

Álvarez, P. (2008). "El Modelo de Rasch como herramienta para obtener una única prioridad entre varias". En J. Febles Acosta (Coord.), "Los Modelos de Rasch en Administración de Empresas. Aplicaciones Avanzadas", 25-38. Santa Cruz de Tenerife: Fundación FYDE Caja Canarias.

Andersen, E. B. (1973). "A goodness of fit test for the Rasch Model”. Psychometrika, 38 (1), 123-140. https://doi.org/10.1007/BF02291180

Andrich, D. (1978). "A rating formulation for ordered response categories". Psychometrika, 43, 357-374. https://doi.org/10.1007/BF02293814 
Andrich, D. (1988a). "A General Form of Rasch's Extended Logistic Model for Partial Credit Credit Scoring". Applied Measurement in Education, 1 (4), 363-378. https://doi. org/10.1207/s15324818ame0104 7

Andrich, D. (1988b). "Rasch Models for measurement". Newbury Park, California: Sage. https://doi.org/10.4135/9781412985598

Argoti, A. C. (2009). "Las pymes agroindustriales de Nariño y su aporte en el desarrollo de la región". Tendencias, 10 (1, 2009), 7-34.

Badenes Plá, N. (2009). "El Modelo de Rasch aplicado a la medición multidimensional de la pobreza en España". XVI Encuentro de Economía Pública: 5 y 6 de febrero de 2009: Palacio de Congresos de Granada.

Cliff, N. (1992). "Abstract measurement theory and the revolution that never happened". Psychological Science, 3 (3), 186-190. https://doi.org/10.1111/j.1467-9280.1992. tb00024.x

Escalona, A. I., Loscertales, B. y Climent, E. A. (2011). "Convenciones, prácticas empresariales y pautas para el desarrollo económico y territorial: Las industrias del vino y del calzado en la provincia de Zaragoza". Anales de geografía de la Universidad Complutense, 31 (2), 77-102. https://doi.org/10.5209/rev_AGUC.2011.v31.n1.37023

Febles, J. (2008). "Los Modelos de Rasch en Administración de EmpreSAs: Aplicaciones Avanzadas". Santa Cruz de Tenerife: Fundación FYDE Caja Canarias.

Galindo Pérez de Azpillaga, L. (2008). "Desarrollo territorial en la Vega Media de Sevilla: Elementos de un tejido empreSArial urbano-rural". Revista de estudios andaluces, 27, 155-203. https://doi.org/10.12795/rea.2008.i27.06

Linacre, J. M. (2014). “WINSTEPS®. Rasch Measurement Computer Program User's Guide”. Beaverton, Oregon: Winsteps. com. (Versión 3.81.0).

Lord, F. M. (1953). "On the Statistical Treatment of Football Numbers". American Psychologist, 8, 750-751. https://doi.org/10.1037/h0063675

Masters, G. N. y Wright, B. D. (1984). "The essential process in a family of measurement models". Psychometrika, 49, 529-544. https://doi.org/10.1007/BF02302590

Melgarejo, Z., Vera-Colina, M. A. y Mora-Riapira, E. (2013). "Competitividad de la Mipyme y deSArrollo regional: estudio del caso colombiano". Tendencias, 14 (2), 184-215.

Michell, J. (1999). "Measurement in psychology: a critical history of a methodological concept”. Cambridge (UK): Cambridge University Press. https://doi.org/10.1017/ CBO9780511490040

Nunes, O. M. y Karnopp, E. (2015). “As Potencialidades Endógenas do Desenvolvimento Regional. Estudo de Caso do Município de Júlio de Castilhos/RS". Desenvolvimento em questao, Editora Unijuí, 13 (30), abr./jun., 203-229. https://doi. org/10.21527/2237-6453.2015.30.203-229

Oreja, J. R. (2005). "Introducción a la medición objetiva en Economía, Administración y Dirección de Empresas: El Modelo de Rasch". IUDE. Serie Estudios, 2005/47. Santa Cruz de Tenerife: Instituto Universitario de la Empresa (IUDE) de la Universidad de La Laguna. Consultada el 28 de enero de 2018, en: http://iude.webs.ull.es/investigacion/ publicaciones/pdf_docs_trabajo/iude-0547.pdf

Oreja, J. R. (2015). “Mediciones, posicionamientos y diagnósticos competitivos”. Colección E-book n ${ }^{\circ}$ 9. Instituto Universitario de la Empresa (IUDE) de la Universidad de La Laguna. Santa Cruz de Tenerife: Fundación FYDE Caja Canarias.

Pérez-Conde, Chavarría-Ortiz y Morán-Álvarez (2020). "Comparison of the determinants of the potential of economic development of the municipalities of Andalusia with population range 15.000-20.000 Inhabitants between the years 2007 and 2012". Revista de Estudios Regionales, (118), 115-147. 
Perline, R., Wright, B. D. y Wainer, H. (1979). "The Rasch Model as an Additive Conjoint Measurement". Applied Psychological Measurement, 3 (2), 237-255. https://doi. org/10.1177/014662167900300213

Plaza, P. et Blanco, B. (2015). "Análisis de los problemas que enfrentan las Pymes Agrícolas para su participación en el desarrollo económico local". Revista Publicando, 2 (5, 2015), 256-264.

Rasch, G. (1980). "Probabilistic Models for Some Intelligence and Attainment Test”. Chicago: The University Chicago Press.

Sánchez, L. y Blanco, B. (2012). "El Modelo de Rasch en Dirección de Operaciones" (Rasch Model in Operations Management). Working Papers on Operations Management, 3 (2), 35-47. https://doi.org/10.4995/wpom.v3i2.1037

Sánchez-Torné, I., Morán-Álvarez, J.et Pérez-López (2020), J. “The importance of corporate social responsibility in achieving high corporate reputation". Corporate Social Responsibility and Environmental Management. https://doi.org/10.1002/csr.1993

Sili, M. (2018). "La acción territorial: una propuesta conceptual y metodológica para su análisis". Revista Brasileira de Estudos Urbanos e Regionais, 20 (1) (jan-abr2018), 11-31. https:// doi.org/10.22296/2317-1529.2018v20n1p11

Silva, I. (2005). "Desarrollo económico local y competitividad territorial en América". Revista de la CEPAL, 85, 81-100. https://doi.org/10.18356/c1a66269-es

Sobrino, J. (2005). "Competitividad territorial: ámbitos e indicadores de Análisis". Economía, sociedad y territorio, 5 (Extra 1, 2005), 123-183.

Stimson, R. J. (2016). "Some Challenges for Regional Science Research". En "New frontiers of regional and urban analysis". Investigaciones Regionales - Journal of Regional Research, 36 (Monográfico 2016), 11-34.

Subiabre, A. Y. y Chacur, A. A. (2006). "Competitividad territorial y estrategia empreSArial: El caso de la industria forestal exportadora de la Región del Bío-Bío". Horizontes empresariales, 5 (1), 39-51.

Torrejón, M., Such, J. y Tomás, J. A. (1996). “Competitividad, estrategia empresarial y territorio: El caso de la industria textil valenciana". Economía industrial, 308, 139-148.

Tristán, A. (2001). “Análisis de Rasch para todos”. México: CENEVAL (Centro Nacional de Evaluación para la Educación Superior, A. C.).

Vázquez Barquero, A. (2012). “La recuperación de la economía española, una cuestión territorial? El papel de las empresas y de los espacios innovadores". Revista de Estudios Empresariales. Segunda época, 1, 5-26. 


\section{ANEXO}

TABLA A1. EMPRESAS SELECCIONADAS DEL MUNICIPIO DE OSUNA

\begin{tabular}{|c|c|c|c|}
\hline 1 & $\begin{array}{l}\text { ACRISTALAMIENTOS VILLA } \\
\text { DUCAL SL. }\end{array}$ & 70 & HIJOS DE ESPUNY SA \\
\hline 2 & AGENCIA DE VIAJES OSUNA SL & 71 & HIPER MULTIPRECIO ORIENTAL S.L \\
\hline 3 & AGIL FORMACION SL & 72 & HOSPEDERIA DEL MONASTERIO SLL. \\
\hline 4 & AGRICOLA FDEZ BORRERO SL & 73 & IBEMA, OBRAS Y SERVICIOS SL. \\
\hline 5 & AGRICOLA LA HEREDAD SL & 74 & IMPORTACION AUTOMOVILES ALVARO SL \\
\hline 6 & $\begin{array}{l}\text { AGRICULTURA Y SERVICIOS EL } \\
\text { ALJIBON SL. }\end{array}$ & 75 & $\begin{array}{l}\text { IMPRENTA LA UNION HERMANOS } \\
\text { BELLIDO SL }\end{array}$ \\
\hline 7 & AGROPECUARIA POZO SANTO SL & 76 & INSTALACIONES ELECTRICAS INELGA SL \\
\hline 8 & ALBARIZUELA 2007 SL & 77 & INVERSIONES CARMONA BEJARANO SL \\
\hline 9 & ALJOSAN S.L. & 78 & JOSE LOPEZ MAZUELOS JOLMA S.L. \\
\hline 10 & $\begin{array}{l}\text { ALMACENES DE LA FUENTE } \\
\text { MARTOS SA }\end{array}$ & 79 & JOSE MARIA AGUILAR MOYA E HIJOS SL \\
\hline 11 & ALVARO ESPUNY S.L. & 80 & JUAN PEÑA DE LA PUERTA SL \\
\hline 12 & ALVARO MORENO SL & 81 & LA DUENA BAJA S.L. \\
\hline 13 & $\begin{array}{l}\text { ARREGUI ELECTRODOMESTICOS } \\
\text { SL }\end{array}$ & 82 & $\begin{array}{l}\text { LARGUI INMOBILIARIA SOCIEDAD } \\
\text { LIMITADA. }\end{array}$ \\
\hline 14 & ARREGUI Y OÑA SERVICIOS SL & 83 & LEGUMBRES JALDON SA \\
\hline 15 & ARTEDOS OSUNA SLL & 84 & LOTERIA LA TIA JULIA SL \\
\hline 16 & AUTOMOTOR OSUNA S.L. & 85 & LUIS PEREZ MARTI E HIJOS SL \\
\hline 17 & AUTOS VILLA DUCAL SL & 86 & MADRE CARMEN SL \\
\hline 18 & BERMUDEZ Y CARO SL & 87 & MANCARDE SL \\
\hline 19 & BOPITER SL & 88 & MASUR AGRICOLA SL \\
\hline 20 & $\begin{array}{l}\text { CARPINTERIA ARTESANAL } \\
\text { ALSAGO SL }\end{array}$ & 89 & MONTORO SAT \\
\hline 21 & CARPINTERIA FADEMA SRL & 90 & MUEBLES ANTONIO MARTIN SL \\
\hline 22 & CECILIA PEREZ ASESORES SLL & 91 & MULTIAUTOS CUEVAS SL \\
\hline 23 & $\begin{array}{l}\text { CELEMI MONTAJES ELECTRICOS } \\
\text { SL }\end{array}$ & 92 & MUÑOZ PEREZ SA \\
\hline 24 & CEMEDI OSUNA SRL & 93 & NUÑEZ TALLER MECANICO SL \\
\hline 25 & $\begin{array}{l}\text { CENTRO DE ESPECIALIDADES } \\
\text { MEDICAS DE OSUNA-CEDEMO SL }\end{array}$ & 94 & OPTICA RODRIGUEZ SL \\
\hline 26 & $\begin{array}{l}\text { CLIMATIZACION Y FRIO } \\
\text { INDUSTRIAL ANTONIO CAMUÑEZ } \\
\text { SL }\end{array}$ & 95 & OSUNA MISSION SOCIEDAD LIMITADA \\
\hline 27 & CLINICA GARCIA HERRAINZ SL & 96 & OSUNA OLIVA SL \\
\hline 28 & CLINICA OSUNA DENTAL SL & 97 & OSUNALIA SOCIEDAD LIMITADA. \\
\hline 29 & COMPAÑIA CAMPOS SA & 98 & OSUNAXXI SOCIEDAD LIMITADA. \\
\hline 30 & $\begin{array}{l}\text { COMPAÑIA OLEICOLA DE } \\
\text { REFINACION Y ENVASADO SA }\end{array}$ & 99 & PANCUEVAS SL \\
\hline 31 & CONFITERIA SAN VALENTIN SLL & 100 & PARAFARMACIA AL GALOPE SL \\
\hline 32 & CONFRAPER SL & 101 & PARALOMELFAR SL \\
\hline 33 & CONSTRU RAMIREZ FUENTES SL & 102 & $\begin{array}{l}\text { PAVIMENTOS ASFALTICOS ANDALUCES } \\
\text { SL }\end{array}$ \\
\hline
\end{tabular}




\begin{tabular}{|c|c|c|c|}
\hline 34 & CONSTRUCCIONES GONYPAL SL & 103 & PAVIURSO SL \\
\hline 35 & $\begin{array}{l}\text { CONSTRUCCIONES } \\
\text { PROFESIONALES DE OSUNA SL }\end{array}$ & 104 & PRADAS Y GUTIERREZ ABOGADOS SL \\
\hline 36 & $\begin{array}{l}\text { CONSTRUCCIONES RUEDA } \\
\text { OSUNA SL }\end{array}$ & 105 & $\begin{array}{l}\text { PREFABRICADOS Y MORTEROS DEL SUR } \\
\text { SL }\end{array}$ \\
\hline 37 & CONSTRUCCIONES SAORFA SRL & 106 & $\begin{array}{l}\text { RAFAEL MARQUINA \& ASOCIADOS SL } \\
\text { PROFESIONAL }\end{array}$ \\
\hline 38 & CONSTRUCCIONES SEYCOSUR SL & 107 & $\begin{array}{l}\text { RC GESTION Y DESARROLLOS DE } \\
\text { PROYECTOS COMERCIALES SL }\end{array}$ \\
\hline 39 & COPISTERIAS DEL SUR SL & 108 & $\begin{array}{l}\text { RED DE ESTACION DE SERVICIOS LAS } \\
\text { VEGAS SL }\end{array}$ \\
\hline 40 & CORTIJO ALGARABEJO S.L. & 109 & $\begin{array}{l}\text { REPUESTOS M CAMUÑEZ SOCIEDAD } \\
\text { LIMITADA. }\end{array}$ \\
\hline 41 & CORTIJO CARRION S.L. & 110 & $\begin{array}{l}\text { REVESTIMIENTOS DE PINTURAS Y } \\
\text { ESPUMAS AISLANTES SL }\end{array}$ \\
\hline 42 & CREACIONES HERYMAT SL & 111 & ROSAN DEMOLICIONES SL \\
\hline 43 & CRISTALERIA OSUNA SL & 112 & S A T LAS TRES PALMERAS \\
\hline 44 & CRISTALURSO SLL & 113 & SAN ARCADIO SL \\
\hline 45 & CRISTOBAL GOMEZ ROJAS SL & 114 & SAN PEDRO 20 S.L. \\
\hline 46 & CUBAS LUIS DOMINGUEZ SL & 115 & SANOR RESTAURA SL. \\
\hline 47 & DANJO OBRAS Y PROYECTOS SL & 116 & SAT N 3253 SAN ARCADIO \\
\hline 48 & DASY ORGANIZACION SL & 117 & SECUEN SLL \\
\hline 49 & $\begin{array}{l}\text { DECORACIONES Y PINTURAS } \\
\text { EGIDO S L }\end{array}$ & 118 & SERVICIOS AGRICOLAS FITOCASH SL. \\
\hline 50 & $\begin{array}{l}\text { DELGADO ORTEGA FRANCISCO } \\
\text { ANTONIO 002375082Z SLNE }\end{array}$ & 119 & SOLANZUR $13 \mathrm{SL}$ \\
\hline 51 & DONJAL SL & 120 & SOLANZUR 14 SL \\
\hline 52 & DONJALFER SL & 121 & SUMINISTROS ELECTRICOS OSUNA SL \\
\hline 53 & EL VILLAR DE LAS CULEBRAS SL & 122 & TALLER ELECTROMECANICA OSUNA SLL \\
\hline 54 & ELECTRODIMAR SL & 123 & TALLERES BLANCOMOTOR SL \\
\hline 55 & $\begin{array}{l}\text { EMPRESARIAL MORON ACTIVA } \\
\text { SL }\end{array}$ & 124 & $\begin{array}{l}\text { TALLERES HERMANOS JIMENEZ REYES } \\
\text { SL }\end{array}$ \\
\hline 56 & $\begin{array}{l}\text { ESYPA SERVICIOS } \\
\text { INMOBILIARIOS SL }\end{array}$ & 125 & TELECOMUNICACION OSUNA SL \\
\hline 57 & EXPLOTACION OXONA S L & 126 & $\begin{array}{l}\text { TODOGANGAS URSO SOCIEDAD } \\
\text { LIMITADA }\end{array}$ \\
\hline 58 & $\begin{array}{l}\text { EXPLOTACIONES RUSTICAS EL } \\
\text { CANTALEJO SL }\end{array}$ & 127 & TURISMO RURAL OSUNA SL \\
\hline 59 & $\begin{array}{l}\text { FERNANDO RODRIGUEZ E HIJOS } \\
\text { SL. }\end{array}$ & 128 & UFAGEST SL \\
\hline 60 & FINCA GOBANTES SL & 129 & $\begin{array}{l}\text { UNION FAMILIAR CONSTRUCTORA } \\
\text { UFACON SL }\end{array}$ \\
\hline 61 & FRANCISCO PERAL SL & 130 & URBANEJA Y ESPINOSA SL \\
\hline 62 & FRIO OSUNA SLL & 131 & URBANIZACION MANCILLA SL \\
\hline 63 & FRUTAS DIAZ SANCHEZ SL & 132 & URSO FINCA SL \\
\hline 64 & FRUTAS HERRERA SL & 133 & $\begin{array}{l}\text { URSO SERVICIOS MEDIOAMBIENTALES } \\
\text { SL }\end{array}$ \\
\hline
\end{tabular}




\begin{tabular}{|l|l|l|l|}
\hline 65 & GARCIA CALDERON SL & 134 & URSO SISTEMAS INFORMATICOS SL \\
\hline 66 & GENITIVA IULIA SL & 135 & URSO SL \\
\hline 67 & GRANJA LA DICHA SL & 136 & VDA DE MANUEL ARREGUI SL \\
\hline 68 & HACIENDA LA BEATA S.L. & 137 & YUROAN SLL \\
\hline 69 & HACIENDA LA CARRASCALA S.L. & \multicolumn{2}{|l|}{ Fuente: Sistema de Análisis de Balances Ibéricos. } \\
\hline
\end{tabular}

TABLA A2. MEDIDA DE FORTALEZA ECONÓMICA EMPRESARIAL DE LAS EMPRESAS DEL MUNICIPIO DE OSUNA

\begin{tabular}{|c|c|c|c|c|c|c|c|}
\hline \multirow{2}{*}{$\begin{array}{c}N^{0} \text { de } \\
\text { entrada }\end{array}$} & \multirow[t]{2}{*}{ Medida } & \multirow{2}{*}{$\begin{array}{l}\text { DS: Desv. } \\
\text { Estándar }\end{array}$} & \multicolumn{2}{|c|}{ INFIT } & \multicolumn{2}{|c|}{ OUTFIT } & \multirow[t]{2}{*}{ Empresa } \\
\hline & & & MNSQ & ZSTD & MNSQ & ZSTD & \\
\hline 30 & 0,62 & 0,30 & 7,46 & 4,7 & 9,90 & 4,6 & $\begin{array}{l}\text { COMPAÑÍA OLEICOLA DE } \\
\text { REFINACIÓN Y } \\
\text { ENVASADO SA }\end{array}$ \\
\hline 95 & 0,25 & 0,26 & 4,43 & 4,8 & 5,69 & 4,2 & OSUNA MISSION SL \\
\hline 69 & 0,06 & 0,26 & 3,53 & 3,7 & 2,82 & 2,4 & $\begin{array}{l}\text { HACIENDA LA CARRASCAL } \\
\text { SL }\end{array}$ \\
\hline 130 & $-0,01$ & 0,26 & 5,23 & 4,8 & 4,28 & 3,5 & URBANEJA Y ESPINOSA SL \\
\hline 97 & $-0,08$ & 0,27 & 1,60 & 1,2 & 1,67 & 1,2 & OSUNALIA SL \\
\hline 11 & $-0,23$ & 0,29 & 1,31 & 0,7 & 1,23 & 0,5 & ALVARO ESPUNY SL \\
\hline 86 & $-0,23$ & 0,29 & 2,06 & 1,6 & 2,03 & 1,4 & MADRE CARMEN SL \\
\hline 129 & $-0,32$ & 0,30 & 2,12 & 1,5 & 1,42 & 0,8 & UFACON SL \\
\hline 48 & $-0,41$ & 0,32 & 2,73 & 1,9 & 4,12 & 2,4 & DASY ORGANIZACIÓN SL \\
\hline 100 & $-0,41$ & 0,32 & 0,44 & $-0,8$ & 0,40 & $-0,6$ & $\begin{array}{l}\text { PARAFARMACIA AL GALOPE } \\
\text { SL }\end{array}$ \\
\hline 135 & $-0,41$ & 0,32 & 0,20 & $-1,5$ & 0,22 & $-1,1$ & URSO SL \\
\hline 6 & $-0,52$ & 0,34 & 0,24 & $-1,2$ & 0,28 & $-0,8$ & $\begin{array}{l}\text { AGRICULTURA Y SERVICIOS } \\
\text { EL ALJIBON SL }\end{array}$ \\
\hline 41 & $-0,52$ & 0,34 & 0,23 & $-1,2$ & 0,21 & $-0,9$ & CORTIJO CARRION SL \\
\hline 88 & $-0,52$ & 0,34 & 0,67 & $-0,2$ & 0,97 & 0,3 & MASUR AGRICOLA SL \\
\hline 112 & $-0,52$ & 0,34 & 0,37 & $-0,8$ & 0,32 & $-0,7$ & SAT LAS TRES PALMERAS \\
\hline 121 & $-0,52$ & 0,34 & 0,37 & $-0,8$ & 0,32 & $-0,7$ & $\begin{array}{l}\text { SUMINISTROS ELECTRICOS } \\
\text { OSUNA SL }\end{array}$ \\
\hline 24 & $-0,64$ & 0,36 & 0,39 & $-0,7$ & 0,27 & $-0,6$ & CEMEDI OSUNA SRL \\
\hline 40 & $-0,64$ & 0,36 & 0,42 & $-0,6$ & 0,35 & $-0,4$ & CORTIJO ALGARABEJO SL \\
\hline 42 & $-0,64$ & 0,36 & 0,54 & $-0,4$ & 0,50 & $-0,2$ & CREACIONES HERYMAT SL \\
\hline 57 & $-0,64$ & 0,36 & 1,79 & 1,0 & 1,35 & 0,7 & EXPLOTACION OXONA SL \\
\hline 70 & $-0,64$ & 0,36 & 1,06 & 0,4 & 1,49 & 0,8 & HIJOS DE ESPUNY SA \\
\hline 81 & $-0,64$ & 0,36 & 0,39 & $-0,7$ & 0,27 & $-0,6$ & LA DUEÑA BAJA SL \\
\hline 89 & $-0,64$ & 0,36 & 0,42 & $-0,6$ & 0,35 & $-0,4$ & MONTORO SAT \\
\hline 92 & $-0,64$ & 0,36 & 0,42 & $-0,6$ & 0,35 & $-0,4$ & MUÑOZ PEREZ SA \\
\hline 107 & $-0,64$ & 0,36 & 0,30 & $-0,9$ & 0,32 & $-0,5$ & $\begin{array}{l}\text { RC GESTION Y } \\
\text { DESARROLLOS DE } \\
\text { PROYECTOS COMERCIALES } \\
\text { SL }\end{array}$ \\
\hline
\end{tabular}




\begin{tabular}{|c|c|c|c|c|c|c|c|}
\hline \multirow{2}{*}{$\begin{array}{l}\mathrm{N}^{0} \mathrm{de} \\
\text { entrada }\end{array}$} & \multirow{2}{*}{ Medida } & \multirow{2}{*}{$\begin{array}{l}\text { DS: Desv. } \\
\text { Estándar }\end{array}$} & \multicolumn{2}{|c|}{ INFIT } & \multicolumn{2}{|c|}{ OUTFIT } & \multirow{2}{*}{ Empresa } \\
\hline & & & MNSQ & ZSTD & MNSQ & ZSTD & \\
\hline 133 & $-0,64$ & 0,36 & 0,39 & $-0,7$ & 0,27 & $-0,6$ & $\begin{array}{l}\text { URSO SERVICIOS } \\
\text { MEDIOAMBIENTALES SL }\end{array}$ \\
\hline 62 & $-0,77$ & 0,43 & 1,94 & 1,1 & 1,56 & ,8 & FRIO OSUNA SLL \\
\hline 1 & $-0,79$ & 0,39 & 0,84 & 0,1 & 0,68 & 0,2 & $\begin{array}{l}\text { ACRISTALAMIENTOS VILLA } \\
\text { DUCAL SL }\end{array}$ \\
\hline 10 & $-0,79$ & 0,39 & 0,76 & 0,0 & 0,82 & 0,3 & $\begin{array}{l}\text { ALMACENES DE LA FUENTE } \\
\text { MARTOS SA }\end{array}$ \\
\hline 12 & $-0,79$ & 0,39 & 0,17 & $-1,2$ & 0,39 & $-0,2$ & ALVARO MORENO SL \\
\hline 15 & $-0,79$ & 0,39 & 3,20 & 1,9 & 2,58 & 1,3 & ARTEDOS OSUNA SLL \\
\hline 39 & $-0,79$ & 0,39 & 0,15 & $-1,3$ & 0,13 & $-0,7$ & COPISTERIAS DEL SUR SL \\
\hline 51 & $-0,79$ & 0,39 & 0,15 & $-1,3$ & 0,13 & $-0,7$ & DONJAL SL \\
\hline 61 & $-0,79$ & 0,39 & 0,15 & $-1,3$ & 0,13 & $-0,7$ & FRANCISCO PERAL SL \\
\hline 64 & $-0,79$ & 0,39 & 0,15 & $-1,3$ & 0,13 & $-0,7$ & FRUTAS HERRERA SL \\
\hline 68 & $-0,79$ & 0,39 & 0,79 & 0,0 & 0,55 & 0,0 & HACIENDA LA BEATA SL \\
\hline 83 & $-0,79$ & 0,39 & 0,15 & $-1,3$ & 0,13 & $-0,7$ & LEGUMBRES JALDÓN SA \\
\hline 102 & $-0,79$ & 0,39 & 0,79 & 0,0 & 0,55 & 0,0 & $\begin{array}{l}\text { PAVIMENTOS ASFALTICOS } \\
\text { ANDALUCES SL }\end{array}$ \\
\hline 110 & $-0,79$ & 0,39 & 3,20 & 1,9 & 2,58 & 1,3 & $\begin{array}{l}\text { REVESTIMIENTOS DE } \\
\text { PINTURAS Y ESPUMAS } \\
\text { AISLANTES SL }\end{array}$ \\
\hline 115 & $-0,79$ & 0,39 & 0,44 & $-0,5$ & 0,42 & $-0,1$ & SANOR RESTAURA SL \\
\hline 128 & $-0,79$ & 0,39 & 0,22 & $-1,0$ & 0,20 & $-0,5$ & UFAGEST SL \\
\hline 5 & $-0,95$ & 0,41 & 0,31 & $-0,7$ & 0,20 & $-0,4$ & AGRICOLA LA HEREDAD SL \\
\hline 14 & $-0,95$ & 0,41 & 0,31 & $-0,7$ & 0,20 & $-0,4$ & $\begin{array}{l}\text { ARREGUI Y OÑA SERVICIOS } \\
\text { SL }\end{array}$ \\
\hline 18 & $-0,95$ & 0,41 & 0,06 & $-1,7$ & 0,09 & $-0,7$ & BERMUDEZ Y CARO SL \\
\hline 19 & $-0,95$ & 0,41 & 0,21 & $-1,0$ & 0,49 & 0,0 & BOPITER SL \\
\hline 25 & $-0,95$ & 0,41 & 2,30 & 1,4 & 1,82 & 0,9 & $\begin{array}{l}\text { CENTRO DE } \\
\text { ESPECIALIDADES MEDICAS } \\
\text { DE OSUNA-CEDEMO SL }\end{array}$ \\
\hline 27 & $-0,95$ & 0,41 & 0,38 & $-0,6$ & 0,31 & $-0,2$ & $\begin{array}{l}\text { CLINICA GARCIA HERRAINZ } \\
\text { SL }\end{array}$ \\
\hline 31 & $-0,95$ & 0,41 & 1,02 & 0,3 & 0,56 & 0,1 & $\begin{array}{l}\text { CONFITERIA SAN VALENTIN } \\
\text { SLL }\end{array}$ \\
\hline 32 & $-0,95$ & 0,41 & 0,14 & $-1,3$ & 0,17 & $-0,5$ & CONFRAPER SL \\
\hline 37 & $-0,95$ & 0,41 & 1,85 & 1,1 & 1,76 & 0,9 & $\begin{array}{l}\text { CONSTRUCCIONES SAORFA } \\
\text { SRL }\end{array}$ \\
\hline 65 & $-0,95$ & 0,41 & 0,31 & $-0,7$ & 0,20 & $-0,4$ & GARCIA CALDERÓN SL \\
\hline 78 & $-0,95$ & 0,41 & 1,02 & 0,3 & 0,56 & 0,1 & $\begin{array}{l}\text { JOSE LOPEZ MAZUELOS } \\
\text { JOLMA SL }\end{array}$ \\
\hline 94 & $-0,95$ & 0,41 & 0,14 & $-1,3$ & 0,17 & $-0,5$ & OPTICA RODRIGUEZ SL \\
\hline 104 & $-0,95$ & 0,41 & 0,06 & $-1,7$ & 0,09 & $-0,7$ & $\begin{array}{l}\text { PRADAS Y GUTIERREZ } \\
\text { ABOGADOS SL }\end{array}$ \\
\hline 109 & $-0,95$ & 0,41 & 0,24 & $-1,0$ & 0,20 & $-0,4$ & REPUESTOS M. CAMÚÑEZ SL \\
\hline
\end{tabular}




\begin{tabular}{|c|c|c|c|c|c|c|c|}
\hline \multirow{2}{*}{$\begin{array}{c}\mathrm{N}^{0} \text { de } \\
\text { entrada }\end{array}$} & \multirow[t]{2}{*}{ Medida } & \multirow{2}{*}{$\begin{array}{l}\text { DS: Desv. } \\
\text { Estándar }\end{array}$} & \multicolumn{2}{|c|}{ INFIT } & \multicolumn{2}{|c|}{ OUTFIT } & \multirow[t]{2}{*}{ Empresa } \\
\hline & & & MNSQ & ZSTD & MNSQ & ZSTD & \\
\hline 125 & $-0,95$ & 0,41 & 1,02 & 0,3 & 0,56 & 0,1 & $\begin{array}{l}\text { TELECOMUNICACION } \\
\text { OSUNA SL }\end{array}$ \\
\hline 2 & $-1,13$ & 0,43 & 0,12 & $-1,2$ & 0,10 & $-0,7$ & $\begin{array}{l}\text { AGENCIA DE VIAJES OSUNA } \\
\text { SL }\end{array}$ \\
\hline 7 & $-1,13$ & 0,43 & 0,62 & $-0,1$ & 0,33 & $-0,2$ & $\begin{array}{l}\text { AGROPECUARIA POZO } \\
\text { SANTO SL }\end{array}$ \\
\hline 9 & $-1,13$ & 0,43 & 0,12 & $-1,2$ & 0,10 & $-0,7$ & ALJOSAN SL \\
\hline 16 & $-1,13$ & 0,43 & 0,13 & $-1,2$ & 0,12 & $-0,6$ & AUTOMOTOR OSUNA SL \\
\hline 17 & $-1,13$ & 0,43 & 1,73 & 1,0 & 0,88 & 0,4 & AUTOS VILLA DUCAL SL \\
\hline 23 & $-1,13$ & 0,43 & 0,19 & $-1,0$ & 0,27 & $-0,3$ & $\begin{array}{l}\text { CELEMI MONTAJES } \\
\text { ELECTRICOS SL }\end{array}$ \\
\hline 26 & $-1,13$ & 0,43 & 0,12 & $-1,2$ & 0,10 & $-0,7$ & $\begin{array}{l}\text { CLIMATIZACION Y FRIO } \\
\text { INDUSTRIAL ANTONIO } \\
\text { CAMÚÑEZ SL }\end{array}$ \\
\hline 28 & $-1,13$ & 0,43 & 0,13 & $-1,2$ & 0,12 & $-0,6$ & CLINICA OSUNA DENTAL SL \\
\hline 29 & $-1,13$ & 0,43 & 0,12 & $-1,2$ & 0,10 & $-0,7$ & COMPAÑÍA CAMPOS SA \\
\hline 34 & $-1,13$ & 0,43 & 0,12 & $-1,2$ & 0,10 & $-0,7$ & $\begin{array}{l}\text { CONSTRUCCIONES } \\
\text { GONYPAL SL }\end{array}$ \\
\hline 36 & $-1,13$ & 0,43 & 0,62 & $-0,1$ & 0,33 & $-0,2$ & $\begin{array}{l}\text { CONSTRUCCIONES RUEDA } \\
\text { OSUNA SL }\end{array}$ \\
\hline 43 & $-1,13$ & 0,43 & 0,13 & $-1,2$ & 0,12 & $-0,6$ & CRISTALERIA OSUNA SL \\
\hline 45 & $-1,13$ & 0,43 & 0,62 & $-0,1$ & 0,33 & $-0,2$ & $\begin{array}{l}\text { CRISTOBAL GOMEZ ROJAS } \\
\text { SL }\end{array}$ \\
\hline 52 & $-1,13$ & 0,43 & 0,13 & $-1,2$ & 0,12 & $-0,6$ & DONJALFER SL \\
\hline 56 & $-1,13$ & 0,43 & 0,19 & $-1,0$ & 0,27 & $-0,3$ & $\begin{array}{l}\text { ESYPA SERVICIOS } \\
\text { INMOBILIARIOS SL }\end{array}$ \\
\hline 59 & $-1,13$ & 0,43 & 0,62 & $-0,1$ & 0,33 & $-0,2$ & $\begin{array}{l}\text { FERNANDO RODRÍGUEZ E } \\
\text { HIJOS SL }\end{array}$ \\
\hline 60 & $-1,13$ & 0,43 & 0,31 & $-0,7$ & 0,36 & $-0,1$ & FINCA GOBANTES SL \\
\hline 63 & $-1,13$ & 0,43 & 1,36 & 0,7 & 2,47 & 1,2 & FRUTAS DÍAZ SANCHEZ SL \\
\hline 73 & $-1,13$ & 0,43 & 0,13 & $-1,2$ & 0,12 & $-0,6$ & $\begin{array}{l}\text { IBEMA, OBRAS Y SERVICIOS } \\
\text { SL }\end{array}$ \\
\hline 75 & $-1,13$ & 0,43 & 0,12 & $-1,2$ & 0,10 & $-0,7$ & $\begin{array}{l}\text { IMPRENTA LA UNIÓN } \\
\text { HERMANOS BELLIDO SL }\end{array}$ \\
\hline 76 & $-1,13$ & 0,43 & 0,12 & $-1,2$ & 0,10 & $-0,7$ & $\begin{array}{l}\text { INSTALACIONES } \\
\text { ELECTRICAS INELGA SL }\end{array}$ \\
\hline 79 & $-1,13$ & 0,43 & 0,12 & $-1,2$ & 0,10 & $-0,7$ & $\begin{array}{l}\text { JOSE MARIA AGUILAR } \\
\text { MOYA E HIJOS SL }\end{array}$ \\
\hline 80 & $-1,13$ & 0,43 & 0,13 & $-1,2$ & 0,12 & $-0,6$ & $\begin{array}{l}\text { JUAN PEÑA DE LA PUERTA } \\
\text { SL }\end{array}$ \\
\hline 106 & $-1,13$ & 0,43 & 0,12 & $-1,2$ & 0,10 & $-0,7$ & $\begin{array}{l}\text { RAFAEL MARQUINA } \\
\text { \& ASOCIADOS SL } \\
\text { PROFESIONAL } \\
\end{array}$ \\
\hline 108 & $-1,13$ & 0,43 & 0,13 & $-1,2$ & 0,12 & $-0,6$ & $\begin{array}{l}\text { RED DE ESTACIÓN DE } \\
\text { SERVICIOS LAS VEGAS SL }\end{array}$ \\
\hline
\end{tabular}




\begin{tabular}{|c|c|c|c|c|c|c|c|}
\hline \multirow{2}{*}{$\begin{array}{l}\mathrm{N}^{0} \mathrm{de} \\
\text { entrada }\end{array}$} & \multirow[t]{2}{*}{ Medida } & \multirow{2}{*}{$\begin{array}{l}\text { DS: Desv. } \\
\text { Estándar }\end{array}$} & \multicolumn{2}{|c|}{ INFIT } & \multicolumn{2}{|c|}{ OUTFIT } & \multirow[t]{2}{*}{ Empresa } \\
\hline & & & MNSQ & ZSTD & MNSQ & ZSTD & \\
\hline 118 & $-1,13$ & 0,43 & 0,19 & $-1,0$ & 0,27 & $-0,3$ & $\begin{array}{l}\text { SERVICIOS AGRÍCOLAS } \\
\text { FITOCASH SL }\end{array}$ \\
\hline 131 & $-1,13$ & 0,43 & 1,00 & 0,3 & 1,25 & 0,6 & $\begin{array}{l}\text { URBANIZACION MANCILLA } \\
\text { SL }\end{array}$ \\
\hline 136 & $-1,13$ & 0,43 & 0,12 & $-1,2$ & 0,10 & $-0,7$ & $\begin{array}{l}\text { VDA DE MANUEL ARREGUI } \\
\text { SL }\end{array}$ \\
\hline 3 & $-1,32$ & 0,44 & 0,05 & $-1,4$ & 0,07 & $-0,8$ & AGIL FORMACIÓN SL \\
\hline 20 & $-1,32$ & 0,44 & 0,37 & $-0,5$ & 0,52 & 0,1 & $\begin{array}{l}\text { CARPINTERIA ARTESANAL } \\
\text { ALSAGO SL }\end{array}$ \\
\hline 46 & $-1,32$ & 0,44 & 0,27 & $-0,7$ & 0,17 & $-0,5$ & CUBAS LUIS DOMINGUEZ SL \\
\hline 49 & $-1,32$ & 0,44 & 0,05 & $-1,4$ & 0,07 & $-0,8$ & $\begin{array}{l}\text { DECORACIONES Y } \\
\text { PINTURAS EGIDO SL }\end{array}$ \\
\hline 53 & $-1,32$ & 0,44 & 0,60 & $-0,1$ & 0,46 & 0,0 & $\begin{array}{l}\text { EL VILLAR DE LAS } \\
\text { CULEBRAS SL }\end{array}$ \\
\hline 54 & $-1,32$ & 0,44 & 0,20 & $-0,8$ & 0,47 & 0,0 & ELECTRODIMAR SL \\
\hline 58 & $-1,32$ & 0,44 & 0,05 & $-1,4$ & 0,07 & $-0,8$ & $\begin{array}{l}\text { EXPLOTACIONES RÚSTICAS } \\
\text { EL CANTALEJO SL }\end{array}$ \\
\hline 84 & $-1,32$ & 0,44 & 0,05 & $-1,4$ & 0,07 & $-0,8$ & LOTERIA LA TÍA JULIA SL \\
\hline 85 & $-1,32$ & 0,44 & 0,27 & $-0,7$ & 0,17 & $-0,5$ & $\begin{array}{l}\text { LUIS PÉREZ MARTI E HIJOS } \\
\text { SL }\end{array}$ \\
\hline 91 & $-1,32$ & 0,44 & 0,60 & $-0,1$ & 0,46 & 0,0 & MULTIAUTOS CUEVAS SL \\
\hline 99 & $-1,32$ & 0,44 & 0,27 & $-0,7$ & 0,17 & $-0,5$ & PANCUEVAS SL \\
\hline 116 & $-1,32$ & 0,44 & 0,05 & $-1,4$ & 0,07 & $-0,8$ & SATN 3253 SAN ARCADIO \\
\hline 132 & $-1,32$ & 0,44 & 0,27 & $-0,7$ & 0,17 & $-0,5$ & URSO FINCA SL \\
\hline 4 & $-1,51$ & 0,43 & 0,06 & $-1,4$ & 0,06 & $-0,8$ & $\begin{array}{l}\text { AGRICOLA FDEZ BORRERO } \\
\text { SL }\end{array}$ \\
\hline 22 & $-1,51$ & 0,43 & 0,06 & $-1,4$ & 0,06 & $-0,8$ & $\begin{array}{l}\text { CECILIA PÉREZ ASESORES } \\
\text { SLL }\end{array}$ \\
\hline 35 & $-1,51$ & 0,43 & 0,06 & $-1,4$ & 0,06 & $-0,8$ & $\begin{array}{l}\text { CONSTRUCCIONES } \\
\text { PROFESIONALES DE OSUNA } \\
\text { SL }\end{array}$ \\
\hline 38 & $-1,51$ & 0,43 & 0,16 & $-0,9$ & 0,13 & $-0,6$ & $\begin{array}{l}\text { CONSTRUCCIONES } \\
\text { SEYCOSUR SL }\end{array}$ \\
\hline 44 & $-1,51$ & 0,43 & 0,16 & $-0,9$ & 0,13 & $-0,6$ & CRISTALURSO SLL \\
\hline 66 & $-1,51$ & 0,43 & 0,06 & $-1,4$ & 0,06 & $-0,8$ & GENITIVA IULIA SL \\
\hline 71 & $-1,51$ & 0,43 & 0,06 & $-1,4$ & 0,06 & $-0,8$ & $\begin{array}{l}\text { HIPER MULTIPRECIO } \\
\text { ORIENTAL SL }\end{array}$ \\
\hline 72 & $-1,51$ & 0,43 & 0,06 & $-1,4$ & 0,06 & $-0,8$ & $\begin{array}{l}\text { HOSPEDERIA DEL } \\
\text { MONASTERIO SLL }\end{array}$ \\
\hline 98 & $-1,51$ & 0,43 & 0,06 & $-1,4$ & 0,06 & $-0,8$ & OSUNAXXI SL \\
\hline 101 & $-1,51$ & 0,43 & 0,16 & $-0,9$ & 0,13 & $-0,6$ & PARALOMELFAR SL \\
\hline 103 & $-1,51$ & 0,43 & 0,16 & $-0,9$ & 0,13 & $-0,6$ & PAVIURSO SL \\
\hline 117 & $-1,51$ & 0,43 & 0,06 & $-1,4$ & 0,06 & $-0,8$ & SECUEN SLL \\
\hline 119 & $-1,51$ & 0,43 & 0,16 & $-0,9$ & 0,13 & $-0,6$ & SOLANZUR $13 \mathrm{SL}$ \\
\hline 120 & $-1,51$ & 0,43 & 0,16 & $-0,9$ & 0,13 & $-0,6$ & SOLANZUR 14 SL \\
\hline
\end{tabular}




\begin{tabular}{|c|c|c|c|c|c|c|c|}
\hline \multirow{2}{*}{$\begin{array}{l}\mathrm{N}^{0} \text { de } \\
\text { entrada }\end{array}$} & \multirow[t]{2}{*}{ Medida } & \multirow{2}{*}{$\begin{array}{l}\text { DS: Desv. } \\
\text { Estándar }\end{array}$} & \multicolumn{2}{|c|}{ INFIT } & \multicolumn{2}{|c|}{ OUTFIT } & \multirow[t]{2}{*}{ Empresa } \\
\hline & & & MNSQ & ZSTD & MNSQ & ZSTD & \\
\hline 127 & $-1,51$ & 0,43 & 1,86 & 1,0 & 1,30 & 0,7 & TURISMO RURAL OSUNA SL \\
\hline 137 & $-1,51$ & 0,43 & 0,06 & $-1,4$ & 0,06 & $-0,8$ & YUROAN SLL \\
\hline 33 & $-1,68$ & 0,40 & 0,34 & $-0,6$ & 0,23 & $-0,3$ & $\begin{array}{l}\text { CONSTRU RAMIREZ } \\
\text { FUENTES SL }\end{array}$ \\
\hline 74 & $-1,68$ & 0,40 & 0,48 & $-0,4$ & 0,33 & $-0,2$ & $\begin{array}{l}\text { IMPORTACION } \\
\text { AUTOMOVILES ALVARO SL }\end{array}$ \\
\hline 90 & $-1,68$ & 0,40 & 1,90 & 1,1 & 1,21 & 0,6 & $\begin{array}{l}\text { MUEBLES ANTONIO MARTÍN } \\
\text { SL }\end{array}$ \\
\hline 96 & $-1,68$ & 0,40 & 0,09 & $-1,4$ & 0,06 & $-0,8$ & OSUNA OLIVA SL \\
\hline 111 & $-1,68$ & 0,40 & 0,34 & $-0,6$ & 0,23 & $-0,3$ & ROSAN DEMOLICIONES SL \\
\hline 123 & $-1,68$ & 0,40 & 0,48 & $-0,4$ & 0,33 & $-0,2$ & $\begin{array}{l}\text { TALLERES BLANCOMOTOR } \\
\text { SL }\end{array}$ \\
\hline 21 & $-1,83$ & 0,37 & 0,77 & $-0,1$ & 0,45 & 0,0 & CARPINTERIA FADEMA SRL \\
\hline 47 & $-1,83$ & 0,37 & 0,29 & $-1,0$ & 0,14 & $-0,5$ & $\begin{array}{l}\text { DANJO OBRAS Y } \\
\text { PROYECTOS SL }\end{array}$ \\
\hline 67 & $-1,83$ & 0,37 & 6,21 & 3,5 & 3,58 & 1,6 & GRANJA LA DICHA SL \\
\hline 105 & $-1,83$ & 0,37 & 0,78 & 0,0 & 1,80 & 0,9 & $\begin{array}{l}\text { PREFABRICADOS Y } \\
\text { MORTEROS DEL SUR SL }\end{array}$ \\
\hline 13 & $-1,97$ & 0,36 & 0,84 & 0,0 & 0,76 & 0,3 & $\begin{array}{l}\text { ARREGUI } \\
\text { ELECTRODOMÉSTICOS SL }\end{array}$ \\
\hline 55 & $-2,09$ & 0,35 & 4,35 & 3,3 & 2,70 & 1,3 & $\begin{array}{l}\text { EMPRESARIAL MORÓN } \\
\text { ACTIVA SL }\end{array}$ \\
\hline 77 & $-2,09$ & 0,35 & 1,89 & 1,4 & 3,07 & 1,4 & $\begin{array}{l}\text { INVERSIONES CARMONA } \\
\text { BEJARANO SL }\end{array}$ \\
\hline 93 & $-2,09$ & 0,35 & 2,81 & 2,2 & 2,81 & 1,3 & $\begin{array}{l}\text { NÚÑEZ TALLER MECÁNICO } \\
\text { SL }\end{array}$ \\
\hline 126 & $-2,09$ & 0,35 & 1,13 & 0,4 & 0,46 & 0,0 & TODOGANGAS URSO SL \\
\hline 8 & $-2,22$ & 0,35 & 1,43 & 0,8 & 0,75 & 0,3 & ALBARIZUELA 2007 SL \\
\hline 50 & $-2,22$ & 0,35 & 4,26 & 3,3 & 4,39 & 1,8 & $\begin{array}{l}\text { DELGADO ORTEGA } \\
\text { FRANCISCO ANTONIO } \\
002375082 Z \text { SLNE }\end{array}$ \\
\hline 82 & $-2,22$ & 0,35 & 3,13 & 2,5 & 2,88 & 1,4 & LARGUI INMOBILIARIA SL \\
\hline 87 & $-2,22$ & 0,35 & 1,75 & 1,2 & 0,70 & 0,2 & MANCARDE SL \\
\hline 134 & $-2,34$ & 0,37 & 3,64 & 2,6 & 2,81 & 1,4 & $\begin{array}{l}\text { URSO SISTEMAS } \\
\text { INFORMÁTICOS SL }\end{array}$ \\
\hline MEAN & $-1,11$ & 0,40 & 0,84 & $-0,3$ & 0,76 & 0,0 & \\
\hline S.D. & 0,52 & 0,04 & 1,27 & 1,4 & 1,29 & 1,0 & \\
\hline
\end{tabular}

Fuente: Elaboración propia a partir de Winsteps 3.81.0. 